\title{
Low Peclet number flow of a reacting mixture past an array of catalytic wires
}

\author{
Marcos Vera
}

Amable Liñán

We describe the diffusion controlled combustion of a reacting mixture flowing past a two-dimensional array of catalytic wires, as a simple model for the flow through catalytic wire-meshes, of interest for small scale combustion devices.

In order to minimize the reactant leakage when using only a few layers of wires, the Peclet number based on the upstream velocity, $U$, the spacing between the wires, $l$, and the thermal diffusivity of the mixture, $D_{\mathrm{T}}$, must be $P e=U l / D_{\mathrm{T}} \ll 1$. Then, the flow field presents two distinct regions: a convective-diffusive region upstream of the wires, of scale $l / P e$, and a reactive-diffusive region near the wires, of thickness of order $l$.

Assuming first that the gas phase homogeneous reactions are frozen, the analysis provides the dependence of the downstream values of the temperature and the reactant mass fractions on the Peclet number, the Lewis number of the reactants, the temperature of the wires, and the ratio of the wire radius to the wire spacing. The analysis is then generalized to include the effect of the homogeneous reactions, showing that the rate of fuel consumption in the gas phase grows with the ratio of the adiabatic laminar flame velocity to the upstream flow velocity, the product of the Zeldovich number and the Peclet number, and the nondimensional temperature of the wires.

\section{Introduction}

The recent interest in the development of small autonomous power sources for microelectromechanical systems (MEMS), portable electronic devices, etc has led to the development of so called microtechnology based energy and chemical systems (MECS or PowerMEMS), aiming to provide longer power source lifetimes and a reduction in the size, weight and cost of the power generating equipment. 
Due to the high energy densities of hydrocarbon fuels, up to 100 times higher than stateof-the-art lithium-ion batteries, in the last few years there has been a growing interest in the development of small scale combustion devices [1-4]. However, it is well known that scaled-down versions of conventional combustion engines experience several fundamental and technical problems $[1,5]$. From a fundamental point of view, the shorter residence times associated with the small scale of the system together with the longer chemical times induced by the temperature drop associated with the higher surface/volume (i.e. heat losses/production) ratio lead to low values of the Damkhöler number, which may prevent the onset of combustion reactions, leading to the generation of unburned fuel and $\mathrm{CO}$ emissions. These difficulties, together with additional technological problems, have motivated alternative approaches such as heat-recirculating burners [6-9] and/or catalytic micro-reactors $[2,10-13]$.

Catalytic micro-reactors rely on the use of catalytic materials such as noble metal alloys to accelerate the combustion reactions. These can take place at the catalyst surface even when the gas phase temperature is too low for the homogeneous reactions to occur. Among the advantages of catalytic micro-reactors, we find a simpler combustor design, lower temperatures of operation, and a high flux of reactants to the catalyst surface due to the small size of the system. As a consequence, catalytic micro-reactors suffer less material constraints and produce less toxic product emissions.

Although the catalytic oxidation of hydrocarbons has been extensively studied for the production of chemicals [14-16] as well as for its potential to enhance combustion reactions [17] and reduce pollutant emissions [18-21], few studies have been carried out in the field of micro-scale power generation. Recent examples are the works of Kyritsis et al [2], presenting an efficient meso-scale combustor for liquid hydrocarbons based on electrostatic spraying and catalytic combustion, and of Maruta et al [3], who analysed the extinction limits of catalytic combustion in micro-channels.

The aim of this paper is to describe the temperature and concentration fields associated with the flow of a reacting mixture past an array of catalytic wires, as a simple model for the flow through catalytic wire-meshes, of interest for the development of small scale combustion devices.

The flow past a row or array of circular cylinders at low Reynolds numbers has been extensively studied in the literature. Tamada and Fujikawa [22] studied the two-dimensional flow past an infinite row of evenly spaced circular cylinders using Oseen's approximation and obtained the drag on each cylinder, shown to be larger than the drag corresponding to an isolated cylinder due to the interference effects associated with the low Reynolds numbers. This flow was also analysed by Miyagi [23] on the basis of the Stokes equations. Hasimoto [24] described the Stokes flow past square arrays of cylinders in terms of Fourier series of fundamental periodic solutions of the Stokes equations to calculate the drag of a single cylinder in the array. More recently, Ayaz and Pedley [25] considered the flow through an infinite array of closely packed cylinders, and Wang [26] proposed a semi-analytical model for the description of the Stokes slip flow through a grid of evenly spaced circular cylinders, obtaining the pressure drop across the grid.

Regarding the problem of heat transfer, Sangani and Acrivos [27] studied the rate of heat transfer from square and hexagonal arrays of uniformly heated cylinders to the surrounding moving gas in the limit of small Reynolds and Peclet numbers, while Wang and Sangani [28] analysed the limit of small Reynolds but large Peclet numbers considering square and random arrays.

In this paper, we consider the flow of a reacting mixture past an array of parallel round catalytic wires of radius $a$ and spacing $l$ between their centrelines lying along the $z$ direction in the plane $x=0$ (as illustrated in figure 1). Let $U$ be the upstream flow velocity, normal 


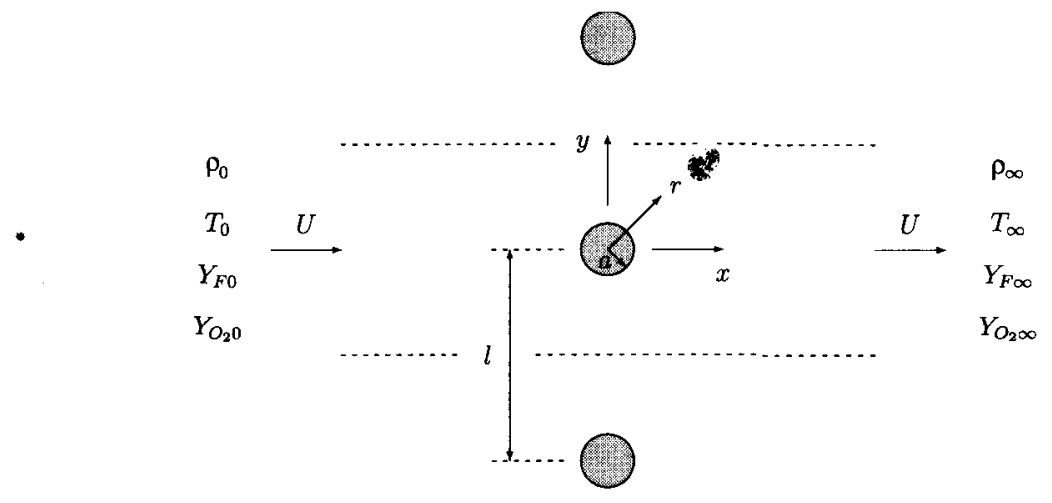

Figure 1. Schematic illustration of the two-dimensional flow of a reacting mixture past a linear array of catalytic wires, showing the coordinates and the upstream and downstream boundary conditions. The basic cell of the flow, repeated infinitely in the transverse direction, is delimited by dashed lines.

to the mesh, of the reacting mixture, and let $T_{0}, \rho_{0}, Y_{\mathrm{F} 0}$ and $Y_{\mathrm{O}_{2} 0}$ be the upstream values of the temperature, the density, and the fuel and oxidizer mass fractions, respectively. We shall assume that the catalytic reaction is so fast that the surface value of the mass fraction of the fuel, considered to be the limiting reactant, is zero.

We shall begin in section 2 with an analysis of the case in which there are no homogeneous reactions in the gas phase. We want to calculate for a given value of the surface temperature of the wire, $T_{\mathrm{s}}$, the downstream values $T_{\infty}, Y_{\mathrm{F} \infty}$ and $Y_{\mathrm{O}_{2} \infty}$ of the temperature and the mass fractions of the mixture. We also want to calculate $q_{1}$, the heat lost by the wire by heat conduction to the gas per unit time and length of the wire, which, under steady and adiabatic conditions, must be equal to the heat release, $Q$, per unit mass of fuel multiplied by the mass consumption rate, $m_{1}$, of the fuel per unit length of the wire. These values will depend on the flow parameters introduced above and, also, on the thermal diffusivity, $D_{\mathrm{T}}$, the Prandtl number, $P r$, and the Lewis numbers, $L_{\mathrm{F}}$ and $L_{\mathrm{O}}$, of the fuel and oxygen. Throughout the analysis, we assume that the heat conductivity of the wire is large enough to ensure that its surface temperature, $T_{\mathrm{s}}$, is uniform.

If the scale, $L$, of the grid is large compared with the mesh cell size, $l$, the flow, as seen with the scale of each cell, appears periodic in the $y$ direction with period $l$. We shall find two main parameters in the nondimensional formulation: the nondimensional wire radius, $\epsilon=a / l<\frac{1}{2}$, and the Peclet number, $P e=U l / D_{\mathrm{T}}$, based on the upstream flow velocity, $U$, normal to the mesh and the thermal diffusivity, $D_{\mathrm{T}}$.

When both $\epsilon$ and $P e$ are of order unity, we may expect the ratio $Y_{\infty}=Y_{\mathrm{F} \infty} / Y_{\mathrm{F} 0}$ to be of order unity, with a significant fraction of the fuel leaking through the mesh. Accordingly, if we want to reduce this leakage, we must either use a mesh with several wire layers or reduce the Peclet number of the flow to values small compared with unity.

After formulating in section 2 the general problem for a mesh with a single or double (staggered) layer of wires, we shall show in section 3 how the problem simplifies when $P e \ll 1$, giving, in particular, closed form analytical expressions in the limiting case when $\epsilon=a / l$ is also small compared with unity. We shall also discuss the effect of changes in density and transport properties with temperature, which in this limiting case can be easily taken into account.

In section 4, we generalize the analysis to include the effect of the homogeneous reactions taking place in the gas phase. We start with the formulation of the problem for arbitrary values 
of the wire temperature, assuming that the activation energy is large, and then discuss the different combustion regimes that arise in the system. In section 5, we present an analysis of the transient effects associated with the time evolution of the wire temperature, and finally, the conclusions are presented in section 6.

\section{Catalytic combustion regime}

When describing the flow structure past meshes with mesh size $l$ small compared with the characteristic large scale size $L$ of the system, one can use homogenization techniques based on the small value of the ratio $l / L$ of the two scales of the system. Thus, for example, the local and surface temperatures of the typically metallic wires will be found to vary, in first approximation, only at distances of the order of the large scale of the system, which are large compared with the scale of the cell.

Accordingly, when describing the flow in a cell, we can use periodic boundary conditions at the cell boundaries. In addition, for simplicity in the presentation, we shall assume that, as is the case in many of the applications, there is no tangential flow velocity upstream of the mesh. Also, for simplicity in the presentation, we shall for the moment neglect the effect of changes in density and transport properties with temperature, although for a small Peclet number they can be easily taken into account.

The Reynolds number $R e=P e / P r$ of the flow will also be considered to be of order unity or smaller than unity, so that the flow is laminar and steady. The gas phase response is then quasi-steady, while unsteady effects are retained only in the description of the transient response of the temperature of the wires. The time variations of $U$ and $T_{\mathrm{s}}$, for example, are considered to occur at times large compared with the residence time, $a / U$, in the cell; the equations will be written in nondimensional form, using the scales $l$ and $U$ for the spatial coordinates and the velocity components. The pressure differences with respect to the upstream pressure will be scaled with $\rho_{0} U^{2}$. For the temperature, we shall use the variable $\Theta=\left(T-T_{0}\right) /\left(T_{\mathrm{e}}-T_{0}\right)$, where $T_{\mathrm{e}}$ is the adiabatic flame temperature given by $Q Y_{\mathrm{F} 0}=c_{\mathrm{p}}\left(T_{\mathrm{e}}-T_{0}\right)$, and for the concentrations we shall define $Y=Y_{\mathrm{F}} / Y_{\mathrm{F} 0}$ and $Y_{\mathrm{O}}=Y_{\mathrm{O}_{2}} / Y_{\mathrm{O}_{2} 0}$. Then the equations take the nondimensional form

$$
\begin{aligned}
& \nabla \cdot v=0 \\
& \operatorname{Pe}\left(\boldsymbol{v} \cdot \nabla v+\nabla p^{\prime}\right)=\operatorname{Pr} \Delta v \\
& \operatorname{Pev} \cdot \nabla \Theta=\Delta \Theta \\
& L_{\mathrm{F}} P e v \cdot \nabla Y=\Delta Y \\
& L_{\mathrm{O}} P e v \cdot \nabla Y_{\mathrm{O}}=\Delta Y_{\mathrm{O}}
\end{aligned}
$$

Three nondimensional transport numbers appear above, the Lewis numbers of the fuel and oxidizer, $L_{\mathrm{F}}=D_{\mathrm{T}} / D_{\mathrm{F}}$ and $L_{\mathrm{O}}=D_{\mathrm{T}} / D_{\mathrm{O}}$, and the Prandtl number, $\operatorname{Pr}=v / D_{\mathrm{T}}$, where $v$ is the kinematic viscosity, $D_{\mathrm{F}}$ and $D_{\mathrm{O}}$ are the binary diffusion coefficients of the reactants and $D_{\mathrm{T}}=k / \rho_{0} c_{\mathrm{p}}$ is the thermal diffusivity of the reacting mixture, defined in terms of the thermal conductivity, $k$, and the specific heat at constant pressure, $c_{p}$.

If we use as coordinates $x$, normal to the mesh, and $y$, parallel to the mesh-defined in figure $2(a)$ for a single layer mesh, and in figure $2(b)$ for a mesh with a double staggered layer-the boundary conditions take the form

$x \rightarrow-\infty: \quad v=i \quad \Theta=Y-1=Y_{\mathrm{O}}-1=p^{\prime}=0$

$x \rightarrow+\infty: \quad v=i \quad \Theta-\Theta_{\infty}=Y-Y_{\infty}=Y_{\mathrm{O}}-Y_{\mathrm{O} \infty}=p^{\prime}-p_{\infty}^{\prime}=0$

where the values $\Theta_{\infty}, Y_{\infty}$ and $Y_{\mathrm{O} \infty}$, and the pressure drop, $-p_{\infty}^{\prime}$, across the mesh, as well as the surface value, $Y_{\mathrm{Os}}$, of the oxygen mass fraction, must be calculated as part of the solution. 
(a) $\rho_{0} \quad \rho_{\infty}$
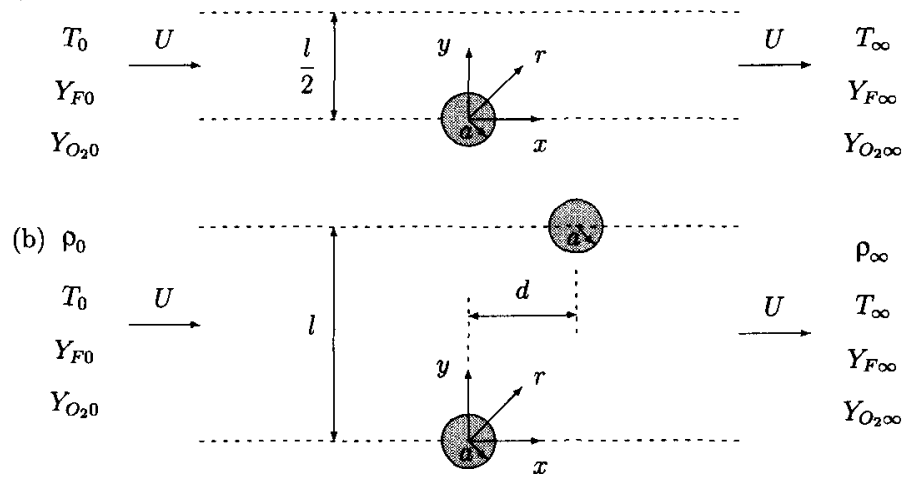

Figure 2. The computational cell, showing the coordinates and the upstream and downstream boundary conditions for $(a)$ linear arrays and $(b)$ double staggered arrays of wires.

In these equations, $v$ is the velocity vector, with components $u$ and $v$ in the $x$ and $y$ directions, respectively, and $i$ denotes the unit vector in the $x$ direction.

The simplified form of the periodicity conditions at the lateral boundaries of each cell is

$$
y= \pm \frac{1}{2}: \quad v=\frac{\partial u}{\partial y}=\frac{\partial \Theta}{\partial y}=\frac{\partial Y}{\partial y}=\frac{\partial Y_{\mathrm{O}}}{\partial y}=0
$$

which are applicable only in the case considered here when there is no transverse flow velocity upstream of the mesh.

We shall only write the boundary conditions for the case $(a)$ of a mesh with a single layer of wires. The formulation of the boundary conditions for the case $(b)$ of a double staggered layer is straightforward; but it should be noted that in the case when both layers of wires have the same temperature, as we shall assume here, it involves the additional parameter $\delta=d / l$ based on the streamwise distance, $d$, between the layers.

The boundary conditions on the surface of the wires are dependent on the overall stoichiometry of the reaction. We shall consider an overall reaction

$$
\mathrm{F}+s \mathrm{O}_{2} \rightarrow(1+s) \text { Products }+(Q)
$$

in which a mass $s$ of oxygen is consumed and an amount $Q$ of heat is released per unit mass of fuel.

At the wire surface, $r=\left(x^{2}+y^{2}\right)^{1 / 2}=\epsilon$, the velocity and the gas phase values of the temperature and mass fraction gradients satisfy the conditions

$$
r=\epsilon: \quad v=\mathbf{0} \quad \Theta-\Theta_{\mathrm{s}}=Y=Y_{\mathrm{O}}-Y_{\mathrm{OS}}=0 \quad \frac{\partial Y}{\partial r}=\frac{1}{\phi} \frac{L_{\mathrm{F}}}{L_{\mathrm{O}}} \frac{\partial Y_{\mathrm{O}}}{\partial r}
$$

where $s Y_{\mathrm{F} 0} / Y_{\mathrm{O}_{2} 0}=\phi$ is the upstream value of the equivalence ratio of the mixture. When using the condition $Y=0$ at $r=\epsilon$, we assume that the catalytic reaction is infinitely fast and that the fuel is the limiting reactant. The last relation in (2.10) results from the requirement that the rates of oxygen and fuel consumption per unit surface are in the stoichiometric ratio $s$.

Coming back to dimensional variables, the time evolution of the wire temperature, $T_{\mathrm{s}}$, must be determined during transients by the equation

$$
\rho_{\mathrm{s}} c_{\mathrm{s}} \pi a^{2} \frac{\mathrm{d} T_{\mathrm{s}}}{\mathrm{d} t}=q_{\mathrm{e}}-q_{1}+Q m_{1}
$$


in terms of the values $\rho_{\mathrm{s}}$ and $c_{\mathrm{s}}$ of the wire density and specific heat, the external energy addition, $q_{\mathrm{e}}$, per unit length and time (sum of the heat lost by radiation and the heat conducted along the wire in the $z$ direction, away from the local interval of unit length), and the value,

$$
q_{1}=-\left.2 \pi a k \overline{\frac{\partial T}{\partial r}}\right|_{r=a}
$$

of the heat lost from the wire by heat conduction to the gas. The last term in (2.11) represents the rate of heat released by the catalytic reaction per unit length of the wire, which is equal to the heat, $Q$, generated by the reaction per unit mass of the reactant multiplied by the mass, $m_{1}$, of the reactant consumed by the catalytic reaction per unit time and length of the wire, with

$$
m_{1}=\left.2 \pi a \rho_{0} Y_{\mathrm{F} 0} \frac{D_{\mathrm{T}}}{\overline{L_{\mathrm{F}}}} \overline{\frac{\partial Y}{\partial r}}\right|_{r=a}
$$

The definitions (2.12) and (2.13) involve the mean values of the radial derivatives of $T$ and $Y$ at the wire surface. When writing equation (2.11), we have considered the temperature to

- be uniform across the wire and a function only of $z$ and $t$, which is well justified for slender metallic rods. When solving the equations for the gas phase, $\Theta_{\mathrm{s}}$ will be considered as known.

For the steady adiabatic response of the wires, the energy conservation equation simplifies to

$$
Q m_{\mathbf{l}}=q_{1}=-\left.2 \pi a k\left(T_{\mathrm{e}}-T_{0}\right) \overline{\frac{\partial \Theta}{\partial r}}\right|_{r=a}
$$

From the conservation equations (2.3)-(2.5), when integrated over the fluid volume between two cross sections upstream and downstream of the mesh, taking into account the boundary conditions (2.6)-(2.8) and (2.10) and the definitions, (2.12) and (2.13), of $q_{1}$ and $m_{1}$, we obtain

$$
m_{1}=\rho_{0} U l Y_{\mathrm{F} 0}\left(1-Y_{\infty}\right)=\frac{m_{\mathrm{O}_{2}}}{s} \quad q_{1}=\rho_{0} U l c_{p}\left(T_{\infty}-T_{0}\right)
$$

which can be written in the nondimensional form

$$
\begin{aligned}
& 1-Y_{\infty}=\frac{1-Y_{\mathrm{O} \infty}}{\phi}=\left.\frac{2 \pi \epsilon}{L_{\mathrm{F}} P e} \overline{\frac{\partial Y}{\partial r}}\right|_{r=\epsilon}=\frac{m_{1}}{m_{10}} \\
& \Theta_{\infty}=-\left.\frac{2 \pi \epsilon}{P e} \overline{\frac{\partial \Theta}{\partial r}}\right|_{r=\epsilon}=\frac{q_{1}}{Q m_{10}}
\end{aligned}
$$

where $m_{10}=\rho_{0} U l Y_{\mathrm{F} 0}$ is the mass flux of fuel convected by the fresh mixture far upstream of the wires. Note that (2.16) and (2.17) are valid under transient nonadiabatic conditions. On the other hand, in the steady adiabatic case, when equation (2.14) is also satisfied, we obtain the additional relation

$$
\Theta_{\infty}=1-Y_{\infty}
$$

In summary, in order to calculate the downstream values $\Theta_{\infty}, Y_{\infty}$ and $Y_{\mathrm{O} \infty}$ of the temperature and concentrations, we must begin by solving equations (2.1) and (2.2) for the pressure and velocity field. Then we can solve equations (2.3) and (2.4) to obtain the temperature and fuel concentration fields. Unless we want to calculate, for example, the surface value, $Y_{\mathrm{Os}}$, of $Y_{\mathrm{O}}$ to ensure that it is positive and thus that the fuel is the limiting reactant, we do not need to solve equation (2.5) even though, if we use the reasonable assumption $L_{O}=1$, we can write

$$
Y_{\mathrm{O}}-Y_{\mathrm{Os}}=\left(1-\frac{\Theta}{\Theta_{\mathrm{s}}}\right)\left(1-Y_{\mathrm{Os}}\right)
$$


small compared with unity. In fact, for meshes with wires not widely spaced, when $a / l=\epsilon$ is not small compared with unity-the case considered in this section-we anticipate that $\psi_{a}=P e \Psi_{a}+\cdots$, where the suspension points denote terms of higher order in Pe. Then, in the mesh region, for $x \sim 1$, we can expand $\psi$ in powers of $P e$ as

$$
\psi(x, y, \epsilon, P e)=P e \Psi(x, y, \epsilon)+\cdots
$$

where $\Psi$ satisfies the equation

$$
\Delta \Psi=0
$$

as derived from (3.1) for $P e \rightarrow 0$, to be solved with the lateral symmetry conditions

$$
y= \pm \frac{1}{2}: \quad \frac{\partial \Psi}{\partial y}=0
$$

and the upstream and downstream conditions

$$
\begin{array}{ll}
x \rightarrow-\infty: & \frac{\partial \Psi}{\partial x}+1=0 \\
x \rightarrow+\infty: & \frac{\partial \Psi}{\partial x}=0
\end{array}
$$

resulting from the matching conditions with (3.8). These conditions are equivalent to $\Psi=-x+\Psi_{a}$ at $x \rightarrow-\infty$ and $\Psi=\Psi_{\infty}$ at $x \rightarrow+\infty$, with $\Psi_{a}(\epsilon)$ and $\Psi_{\infty}(\epsilon)$ to be found as part of the solution. This is uniquely determined when we impose the additional condition at the wire surface:

$$
r=\left(x^{2}+y^{2}\right)^{1 / 2}=\epsilon: \quad \Psi=0
$$

The solution of this problem, obtained numerically in section 3.3.1 for general values of $\epsilon$, yields the downstream value of the temperature,

$$
T_{\infty}-T_{0}=\left(T_{\mathrm{s}}-T_{0}\right)\left(1-P e \Psi_{\infty}+\cdots\right)
$$

as well as the heat lost from the wire by heat conduction to the gas,

$$
q_{1}=\rho_{0} U l c_{p}\left(T_{\mathrm{s}}-T_{0}\right)\left(1-P e \Psi_{\infty}+\cdots\right)
$$

given by the integral relation (2.15) together with (3.15). Here, the terms of order Pe represent the effects of convection, which tends to reduce the heating efficiency of the wire.

In appendix A we give the asymptotic solution of (3.10)-(3.14) in the case of widely spaced wires, $\epsilon \ll 1$. This leads to closed form analytical expressions for $\Psi_{a}(\epsilon)$ and $\Psi_{\infty}(\epsilon)$, given in equations (A.8) and (A.9), respectively.

\subsection{Effect of changes in density and variable transport properties}

When the Peclet number is small and $\epsilon=a / l \sim 1$, as is assumed here, the temperature drop below the wire temperature, $T_{\mathrm{s}}-T$, in the mesh region is small, of order $P e$, when measured with the overall temperature difference, $T_{\mathrm{s}}-T_{0}$. The changes in density and transport properties are then confined to the upstream preheated layer, while, in first approximation, the inner solution remains unchanged if the gas conductivity and the diffusivities are evaluated at the temperature $T_{\mathrm{s}}$. In the preheated region the energy equation, written here in dimensional form, can be integrated once to give

$$
\rho u c_{p}\left(T-T_{s}\right)-k \frac{\partial T}{\partial x}=\rho_{0} U c_{p}\left(T_{0}-T_{\mathrm{s}}\right)
$$

where $\rho u=\rho_{0} U$ from continuity considerations, stating that the sum of the convective and diffusive enthalpy fluxes across the preheated region is constant. 
Then, in the mesh region, where $T_{\mathrm{s}}-T$ is small compared with $T_{\mathrm{s}}-T_{0}$, the slope of the temperature distribution is given, in a first approximation, by

$$
-\left.\frac{\partial \psi}{\partial x}\right|_{x=0}=\frac{\rho_{0} U c_{p}}{k_{\mathrm{s}}}=\frac{k}{k_{\mathrm{s}}} P e=P e_{\mathrm{s}}
$$

where $P e_{\mathrm{s}}$ represents the Peclet number based on the heat conductivity evaluated at the wire temperature, and the subscript $\mathrm{s}$ denotes magnitude evaluated at the temperature $T_{\mathrm{s}}$.

According to this, the temperature distribution, $\psi$, will still be given by (3.9) if we replace $P e$ by the modified Peclet number $P e_{s}$, while the heat lost from the wires by heat conduction to the gas and the downstream value of the temperature will be given, respectively, by equations (3.16) and (3.15) when they are written in terms of $P e_{\mathrm{s}}$.

\subsection{The catalytic combustion regime for low Peclet numbers}

From inspection of the problems verified by $\Theta, Y, Y_{\mathrm{O}}$ and $\psi$, the temperature and concentration fields can be written as follows,

$$
\begin{aligned}
& \frac{\Theta}{\Theta_{\mathrm{s}}}=1-\psi(x, y, \epsilon, P e) \quad Y=\psi\left(x, y, \epsilon, L_{\mathrm{F}} P e\right) \\
& Y_{\mathrm{O}}=1-\phi+\phi \psi\left(x, y, \epsilon, L_{\mathrm{O}} P e\right)
\end{aligned}
$$

in terms of the solution, $\psi(x, y, \epsilon, P e)$, of the heat conduction problem discussed earlier.

Thus, in a first approximation for small values of $P e$, we may write

$$
\begin{aligned}
& \frac{\Theta}{\Theta_{\mathrm{s}}}=1-P e \Psi+\cdots \quad Y=L_{\mathrm{F}} P e \Psi+\cdots \\
& Y_{\mathrm{O}}=1-\phi+\phi L_{\mathrm{O}} P e \Psi+\cdots
\end{aligned}
$$

so that the downstream values of $\Theta, Y$ and $Y_{0}$, which determine the leakage of fuel and oxygen through the wires as well as the temperature far downstream of the mesh, are given in terms of $\Psi_{\infty}$ by the relations

$$
\begin{aligned}
\frac{\Theta_{\infty}}{\Theta_{\mathrm{s}}} & =1-P e \Psi_{\infty}+\cdots \quad Y_{\infty}=L_{\mathrm{F}} P e \Psi_{\infty}+\cdots \\
Y_{\mathrm{O} \infty} & =1-\phi+\phi L_{\mathrm{O}} P e \Psi_{\infty}+\cdots
\end{aligned}
$$

Equations (3.20), together with (2.15)-(2.17), finally provide us with the diffusive fluxes of heat and reactants from and to the wires:

$$
\begin{aligned}
& \frac{q_{1}}{Q m_{10}}=-\left.\frac{2 \pi \epsilon}{P e} \overline{\frac{\partial \Theta}{\partial r}}\right|_{r=\epsilon}=\Theta_{\infty}=\Theta_{\mathrm{s}}\left(1-P e \Psi_{\infty}+\cdots\right) \\
& \frac{m_{1}}{m_{10}}=\left.\frac{2 \pi \epsilon}{L_{\mathrm{F}} P e} \frac{\partial Y}{\partial r}\right|_{r=\epsilon}=1-Y_{\infty}=1-L_{\mathrm{F}} P e \Psi_{\infty}+\cdots
\end{aligned}
$$

3.3.1. Numerical solution for a single layer of wires. The problem that determines $\Psi(x, y, \epsilon)$, given by (3.10) with the boundary conditions (3.11)-(3.14), was solved numerically using a finite volume method with a structured, curvilinear, boundary-fitted grid. The profiles of the solution along $y=0$ and $y= \pm \frac{1}{2}$ are shown in figure 3 for different values of $\epsilon$, figure 4 shows the values of $\Psi_{\infty}$ and $\Psi_{a}$ as a function of $\epsilon$, and figures 5(a) and 6(a) show contour plots of the solution corresponding to $\epsilon^{-1}=3$ and 10 , respectively.

As can be seen, in order to keep the overall diffusive fluxes of heat and reactants from and to the wires constant, the concentration and temperature gradients at the wire surface increase with $\epsilon^{-1}$. According to (3.21), this leads to lower temperatures and higher reactant 


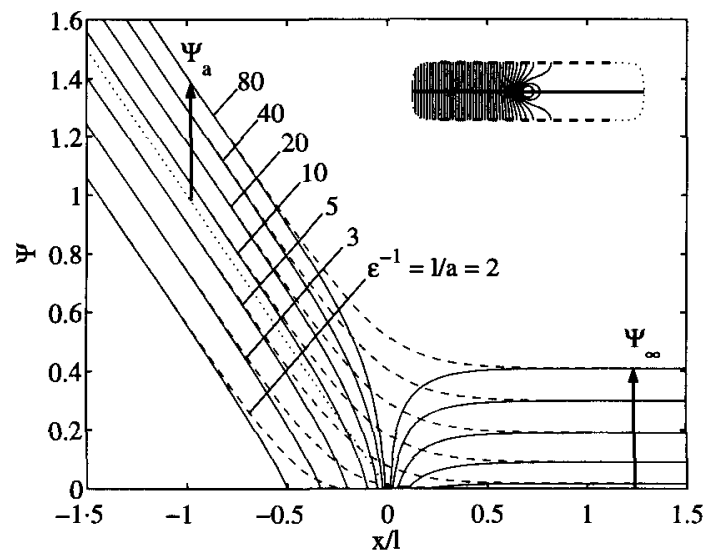

Figure 3. Profiles of $\Psi(x, y)$ along $y=0(-)$ and $y= \pm \frac{1}{2}(---)$ for different values of $\epsilon^{-1}=l / a$.
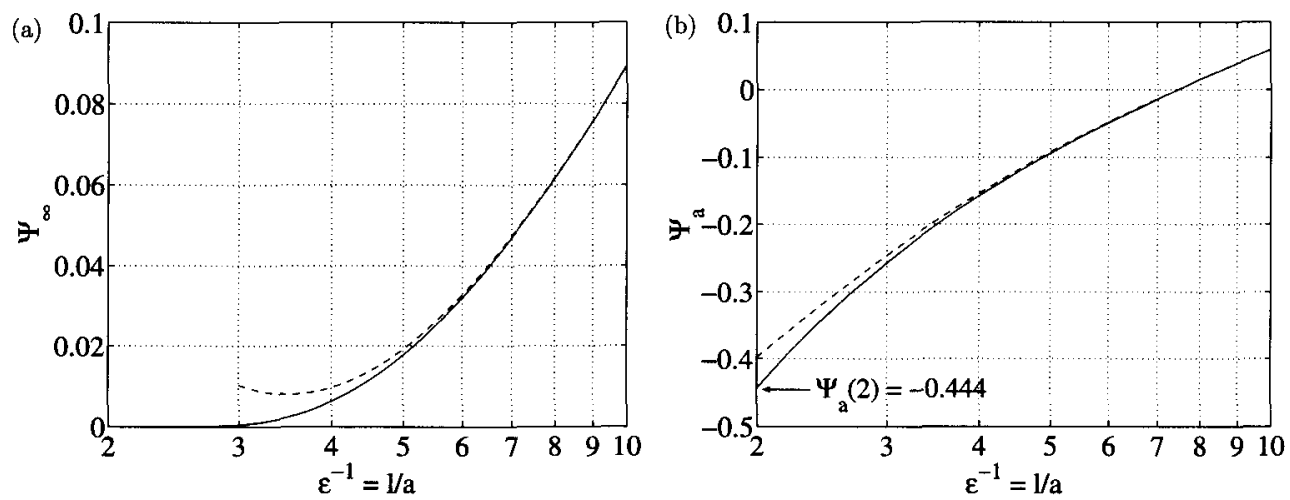

Figure 4. Values of ( $a) \Psi_{\infty}$ and (b) $\Psi_{a}$ as a function of $\epsilon^{-1}=l / a$. The asymptotic predictions for small values of $\epsilon$ given in equations (A.8) and (A.9) are included for comparison (- - - ).

concentrations in the mesh region and downstream of the mesh, as indicated by the large values of $\Psi_{a}$ and $\Psi_{\infty}$ shown in figure 4 for large values of $\epsilon^{-1}$.

Figure 4 also shows that the numerical results are in very good agreement with the asymptotic values of $\Psi_{\infty}$ and $\Psi_{a}$ given by equations (A.8) and (A.9) for small values of $\epsilon$. In particular, the value of $\Psi_{\infty}$ given by (A.8) is seen to fit the computed results to within $1 \%$ for $\epsilon \lesssim \frac{1}{7}$.

3.3.2. Solution for a double staggered layer of wires. In order to analyse the effect of the geometrical configuration on the system's performance, we shall begin by considering the staggered array obtained by moving one cylinder out of each two a distance $d$ downstream from the original grid mid-plane $x=0$. Since the number of wires of the mesh is maintained constant, the overall wire surface area will be constant as well, and the variations of the downstream values of the temperature and reactants mass fractions will be due exclusively to geometric effects.

In general, the temperature of the wires of the second row could be different from that of the wires of the first row, but they should be equal in steady adiabatic conditions. Thus, in 

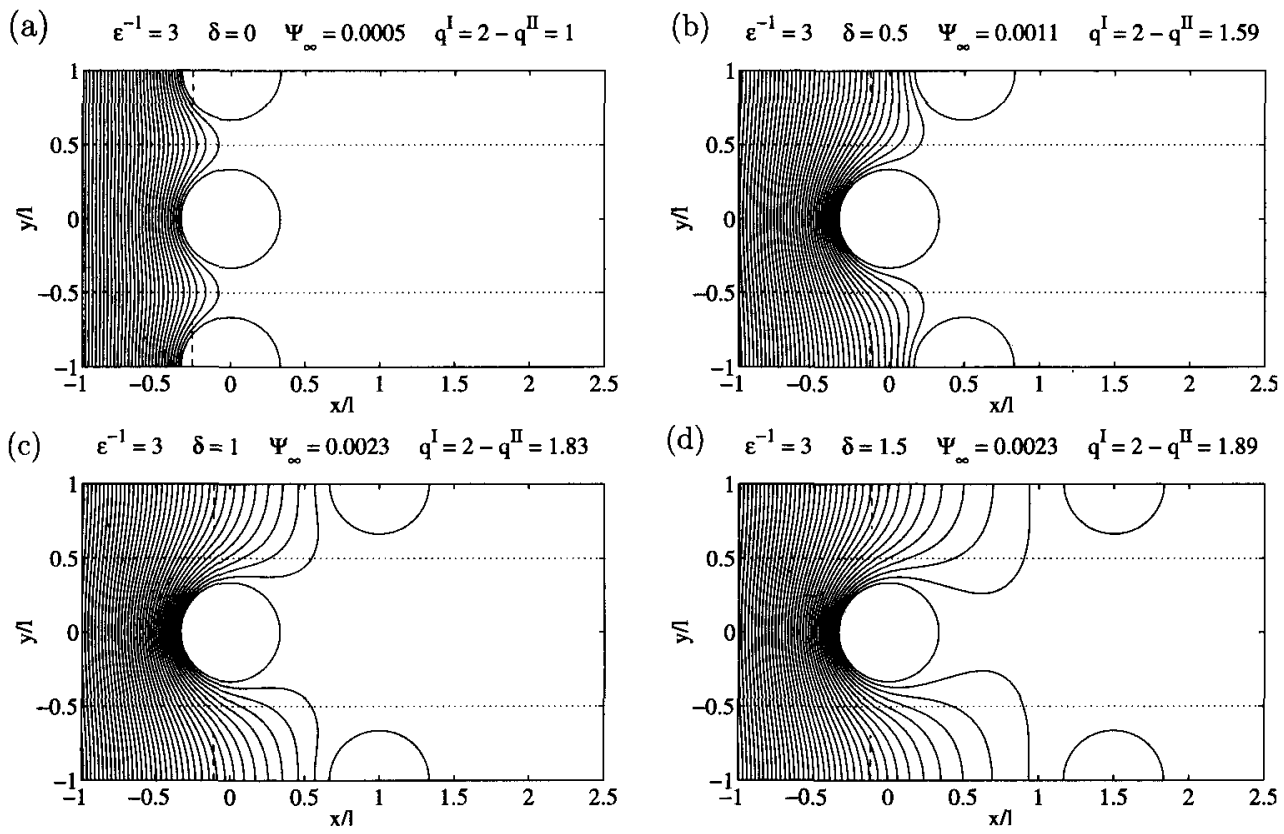

Figure 5. Contour plots of $\Psi$ obtained numerically for $\epsilon^{-1}=l / a=3$ and different values of $\delta=d / l$. The contour lines correspond to increments $\Delta \Psi=0.25(0.025)$ between thick (thin) lines from the $\Psi=0$ value at the wire surface. The corresponding values of $\Psi_{\infty}$ are included in the figures. Dashed lines show the apparent position, $x=\Psi_{a}$, of the iso-contour $\Psi=0$ as seen from $x \rightarrow-\infty$.

what follows, when describing the heat transfer problem, we shall restrict our attention to the case where the temperatures of both rows of wires are identical.

Figures 5 and 6 show contour plots of the solutions corresponding to $\epsilon^{-1}=l / a=3$ and 10 for different values of the staggering distance. As can be seen, for the relatively thick wires of figure 5, the array behaves essentially as an impermeable wall. In this case, the small amount of fuel that leaks through the wires increases with the staggering distance due to the reduction of the obstruction imposed by the wires to the flow as the distance between the wires increases. Note that the obstruction is complete for $\epsilon^{-1}=l / a \leqslant 2$ and $\delta=\left(4 \epsilon^{2}-1\right)^{1 / 2}$, when $\Psi_{\infty}$ should be strictly zero. However, our model could be expected to fail when the nondimensional distance between the cylinders becomes of order $\operatorname{Pe} P \operatorname{Pr}=U l / v \ll 1$ since the local Reynolds number in the narrow gaps between the cylinders is then of order unity.

The obstruction imposed by the wires to the flow is less important for the relatively thin wires shown in figure 6 . In this case the fuel leakage is seen to decrease monotonically with $\delta$, reaching its maximum value in the linear configuration, i.e. for $\delta=0$.

Figure 7 summarizes the results obtained for staggered arrays in terms of $\Psi_{\infty}(\epsilon, \delta)$ as well as the nondimensional mass of reactant consumed at the wires of the first and second rows per unit time and unit length of wire, given, respectively, by

$$
q^{\mathrm{I}}=\frac{q_{1}^{\mathrm{I}}}{Q m_{10}} \quad \text { and } \quad q^{\mathrm{II}}=2-q^{\mathrm{I}}=\frac{q_{1}^{\mathrm{II}}}{Q m_{10}}
$$

In what follows, the superscripts I and II denote quantities corresponding to the first and second rows of wires. Due to the asymmetry of the flow, the mass of reactant consumed at the wires of the first (second) row is larger (smaller) than that consumed by the wires of the linear array. 
(a) $\quad \varepsilon^{-1}=10 \quad \delta=0 \quad \Psi_{\infty}=0.0891 \quad q^{I}=2-q^{I I}=1$

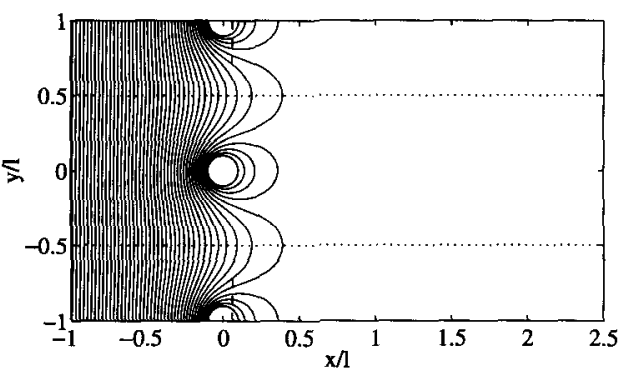

(c) $\varepsilon^{-1}=10 \quad \delta=1 \quad \Psi_{\infty}=0.0742 \quad q^{I}=2-q^{I I}=1.55$

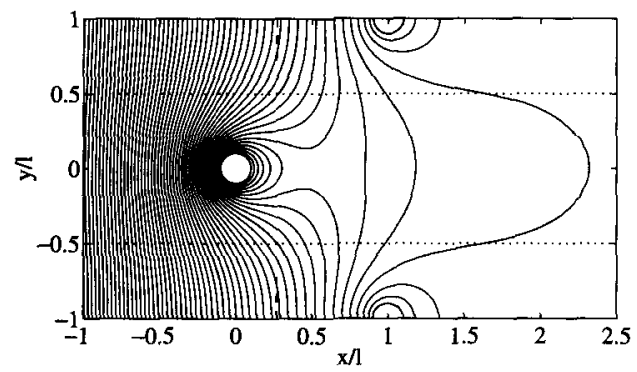

(b) $\quad \varepsilon^{-1}=10 \quad \delta=0.5 \quad \Psi_{\infty}=0.0854 \quad q^{\mathrm{I}}=2-q^{\mathrm{II}}=1.35$

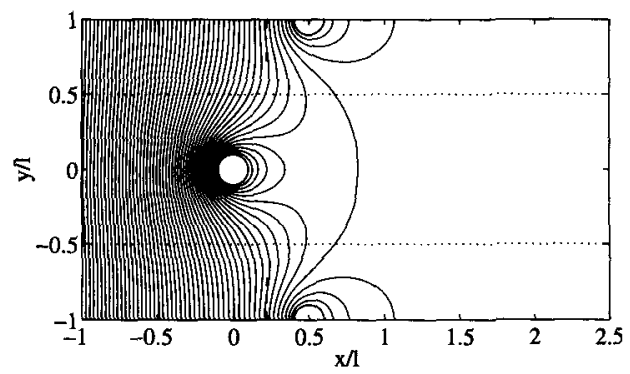

(d) $\quad \varepsilon^{-1}=10 \quad \delta=1.5 \quad \Psi_{\infty}=0.0613 \quad q^{1}=2-q^{I}=1.65$

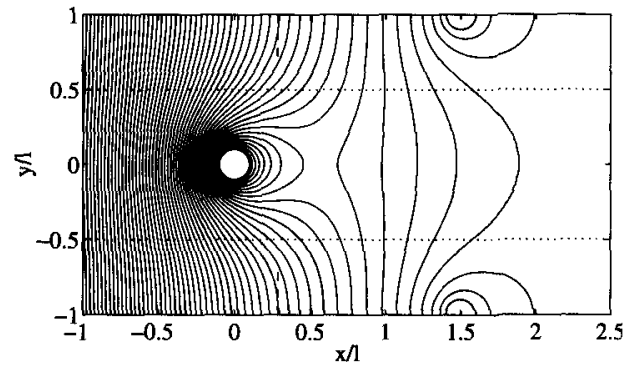

Figure 6. Contour plots of $\Psi$ obtained numerically corresponding to double staggered arrays with $\epsilon^{-1}=l / a=10$ and different values of $\delta=d / l$. The contour lines correspond to increments $\Delta \Psi=0.25(0.025)$ between thick (thin) lines from the $\Psi=0$ value at the wire surface. The corresponding values of $\Psi_{\infty}$ are included in the figures. Dashed lines show the apparent position, $x=\Psi_{a}$, of the iso-contour $\Psi=0$ as seen from $x \rightarrow-\infty$.

Also shown in figure 7 are the asymptotic predictions of the model of appendix A.2 for large values of $\epsilon^{-1}=l / a$. Due to the good agreement with the numerical results observed for staggered arrays, we also present the asymptotic results for the case of in-line arrays sketched in figure $\mathrm{A} 1(\mathrm{c})$, which are limited to large values of both $\epsilon^{-1}=l / a$ and $\epsilon^{-1} \delta=d / a$.

Figure $7(a)$ shows that in-line arrays are less efficient than staggered arrays in terms of minimizing the fuel leakage. However, the differences between the two configurations are seen to vanish for sufficiently large values of $\delta$. This is due to the transverse uniformization of the flow that occurs at distances of order $l$ downstream of the first row. Once the flow is one-dimensional, the lateral translation of the second row is irrelevant.

3.3.3. Effect of the addition of a second row of wires. From an engineering point of view, it is also interesting to analyse the performance of the system after the addition of a second row of wires, i.e. after doubling the number of wires. In particular, we shall consider the staggered configuration shown schematically in figure $8(a)$, which minimizes the fuel leakage as discussed earlier.

After the addition of the second row, the geometrical configuration is defined by the nondimensional parameters $\epsilon^{\prime}=2 a / l=2 \epsilon, \delta^{\prime}=2 d / l$ and $P e^{\prime}=U l / 2 D_{\mathrm{T}}=P e / 2$. Thus, from (3.21), the fuel leakage through the staggered array can be written as

$$
Y_{\infty}^{\mathrm{I}+\mathrm{II}}=L_{\mathrm{F}} P e^{\prime} \Psi\left(\epsilon^{\prime}, \delta^{\prime}\right)=L_{\mathrm{F}} \frac{P e}{2} \Psi\left(2 \epsilon, \delta^{\prime}\right)=\frac{Y_{\infty}^{\mathrm{I}}}{2} \frac{\Psi\left(2 \epsilon, \delta^{\prime}\right)}{\Psi(\epsilon, 0)}
$$

in terms of the fuel leakage $Y_{\infty}^{\mathrm{I}}=L_{\mathrm{F}} P e \Psi(\epsilon, 0)$ through the original linear array. 

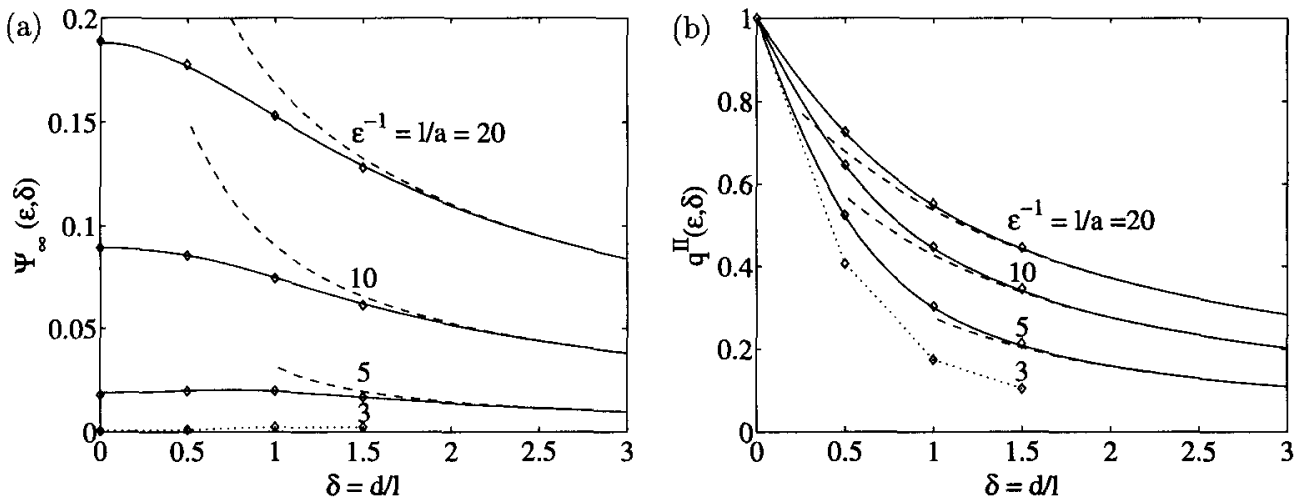

Figure 7. Numerically obtained values of $(a) \Psi_{\infty}$ and (b) rate of fuel consumption per unit length at the second row of wires as a function of $\delta$ for different values of $\epsilon^{-1}(\diamond)$. The asymptotic predictions given by the model of appendix A.2 for staggered arrays (- - and in-line arrays (- - -) are shown for comparison.
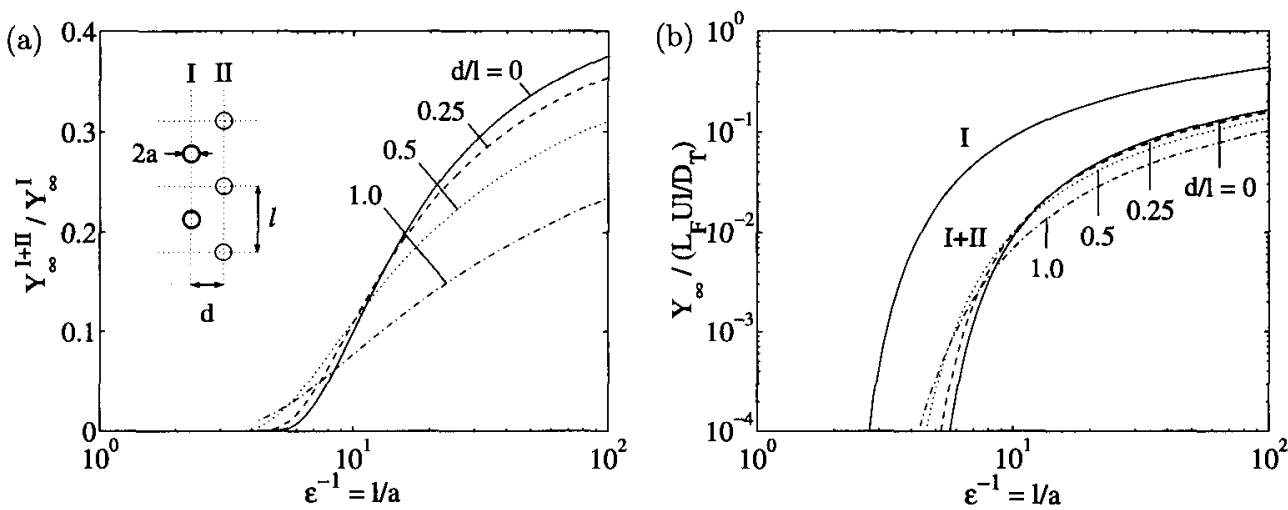

Figure 8. Effect of the addition of a second row of wires on the fuel leakage as a function of $\epsilon^{-1}=l / a$ for various staggering distances: $(a)$ relative reduction of the fuel leakage with respect to its original value; $(b)$ absolute value of the fuel leakage for the single layer (I) and the double staggered layer $(\mathrm{I}+\mathrm{II})$. Note that the case $d / l=0$ corresponds to a linear array with double the number of wires.

Figure 8 shows the downstream value of the reactant mass fraction before and after the addition of the second row of wires for various staggering distances as well as the ratio $Y_{\infty}^{\mathrm{I}+\mathrm{II}} / Y_{\infty}^{\mathrm{I}}$ given by (3.25). As can be seen, the addition of the second row of wires substantially reduces the fuel leakage, which is cut down to less than $10 \%$ for values of $\epsilon^{-1}=l / a \lesssim 10$. However, note that the fuel leakage is a nonmonotonic function of the staggering distance when, for $\epsilon^{-1} \sim 1$, the obstruction effect becomes important.

\section{Effect of the homogeneous reactions}

In this section, we shall generalize the analysis to include the effect of the homogeneous reactions. For definiteness, we shall consider the case of lean mixtures, encountered more frequently in applications [2]. In the analysis, we model the reaction by a one-step irreversible 
reaction of the type (2.9), with an overall Arrhenius reaction rate

$$
\frac{w_{\mathrm{O}_{2}}}{s}=w_{\mathrm{F}}=\rho B Y_{\mathrm{O}_{2}} Y_{\mathrm{F}} \exp \left(-\frac{E}{R T}\right)
$$

with unit reaction orders for both fuel and oxidizer, where for simplicity of presentation we shall ignore the effect of oxygen consumption. However, the analysis will be generalized later to account for this effect for mixtures close to stoichiometric.

\subsection{Formulation of the problem}

When the mixture is lean and such that $0<1-\phi \sim 1$, the effect of the variation of oxygen concentration in the reaction zone, which is of order $\beta_{\mathrm{e}}^{-1}$, can be neglected compared with its final value, $Y_{\mathrm{O} \infty}=1-\phi \sim 1$. Here, $\beta_{\mathrm{e}}=E\left(T_{\mathrm{e}}-T_{0}\right) / R T_{\mathrm{e}}^{2}$ is the Zeldovich numberor nondimensional activation energy - evaluated at the adiabatic flame temperature, which is typically a large number in combustion reactions.

As previously discussed, the distributions of temperature and concentration are no longer one-dimensional for values of $x$ of order $l$. In this region, for $P e \ll 1$, they satisfy in first approximation a reactive-diffusive balance without convection effects. We shall write this balance in nondimensional form by using the scale $l$ for the spatial coordinates and introducing the nondimensional variables $\hat{\Theta}=\left(\Theta-\Theta_{\mathrm{s}}\right) / P e$ and $\hat{Y}=Y /\left(L_{\mathrm{F}} P e\right)$ for the first order perturbations, from the first order values $\Theta=\Theta_{\mathrm{s}}$ and $Y=0$ of the temperature and reactant mass fraction in the mesh region. Thus, we have

$$
\begin{aligned}
& \hat{Y}+\Theta_{\mathrm{s}} \hat{\Theta}=\left(1-\Theta_{\mathrm{s}}\right) \Psi \\
& \Delta \hat{Y}=\bar{U}_{\mathrm{L}}^{2} \exp \left[-\frac{T_{\mathrm{e}}}{T_{\mathrm{s}}} \beta_{\mathrm{e}}\left(1-\Theta_{\mathrm{s}}\right)\right] \frac{b^{2}}{2} \hat{Y} \exp \left(\frac{T_{\mathrm{e}}^{2}}{T_{\mathrm{s}}^{2}} b \Theta_{\mathrm{s}} \hat{\Theta}\right)
\end{aligned}
$$

in the gas domain $r>\epsilon,-\frac{1}{2}<y<\frac{1}{2}$, to be solved with the upstream condition $\hat{Y}=\hat{Y}_{a}-x$ at $x \rightarrow-\infty$, of matching with the upstream solution, the downstream condition $\hat{Y}=\hat{Y}_{\infty}$ at $x \rightarrow+\infty$, and the lateral symmetry condition $\partial \hat{Y} / \partial y=0$ at $y= \pm \frac{1}{2}$. Note that in the reacting case, when $\bar{U}_{\mathrm{L}} \neq 0$, the downstream value of the fuel mass fraction, $\hat{Y}_{\infty}$, must be identically zero, so that using (4.2) gives the downstream temperature as $\hat{\Theta}_{\infty}=\Psi_{\infty}\left(1-\Theta_{\mathrm{s}}\right) / \Theta_{\mathrm{s}}$. Finally, for strongly catalytic wires, we impose the condition that $\hat{Y}=0$ at the wire surface, $r=\epsilon$. Here, the function $\Psi$, which relates $\hat{\Theta}$ and $\hat{Y}$ through equation (4.2), is the solution of the problem given by equation (3.10) with the boundary conditions (3.11)-(3.14) discussed in section 3.1.

The exponential temperature dependence of the reaction rate on the right-hand side of equation (4.3) results from the linearization of the Arrhenius exponent about the temperature of the wires. In addition, we have written the pre-exponential frequency factor, $B$, of the Arrhenius term in terms of the adiabatic laminar flame velocity, $U_{\mathrm{L}}$, of the fresh mixture, given in a first approximation for large values of $\beta_{\mathrm{e}}$ by

$$
U_{\mathrm{L}}^{2}=2 L_{\mathrm{F}} D_{\mathrm{T}} \beta_{\mathrm{e}}^{-2}\left(1-\phi+2 \phi L_{0} \beta_{\mathrm{e}}^{-1}\right) Y_{\mathrm{O}_{2} 0} B \exp \left(-\frac{E}{R T_{\mathrm{e}}}\right)
$$

where in the case of lean mixtures far from stoichiometry, $0<1-\phi \sim 1$, we may neglect the term $2 \phi L_{0} \beta_{\mathrm{e}}^{-1}$ in the bracketed expression. Finally, the ratio of the adiabatic flame temperature to the temperature of the wires appearing in (4.3) can be written in terms of the nondimensional parameters $\Theta_{\mathrm{s}}$ and $\left(T_{\mathrm{e}}-T_{0}\right) / T_{0}=Q Y_{\mathrm{F} 0} / c_{p} T_{0}$ using the relation

$$
\frac{T_{\mathrm{s}}}{T_{\mathrm{e}}}=\Theta_{\mathrm{s}}+\left(1-\Theta_{\mathrm{s}}\right)\left(\frac{T_{0}}{T_{\mathrm{e}}}\right)
$$


Two new parameters appear above in addition to $\Theta_{\mathrm{s}}=\left(T_{\mathrm{s}}-T_{0}\right) /\left(T_{\mathrm{e}}-T_{0}\right), \epsilon=a / l$ and $\left(T_{\mathrm{e}}-T_{0}\right) / T_{0}$, namely the ratio $\bar{U}_{\mathrm{L}}=U_{\mathrm{L}} / U$ of the adiabatic laminar flame velocity to the upstream flow velocity and the product $b=\beta_{\mathrm{e}} P e$ of the Zeldovich number and the Peclet number. Then, $\bar{U}_{\mathrm{L}}$ represents the ratio of the thickness of the convective-diffusive region upstream of the wires, $D_{\mathrm{T}} / U$, to the adiabatic laminar flame thickness, $D_{\mathrm{T}} / U_{\mathrm{L}}$, while the ratio of the wire spacing, $l$, to the characteristic gas phase reaction zone thickness, $\beta_{\mathrm{e}}^{-1} D_{\mathrm{T}} / U_{\mathrm{L}}$, is equal to $b \bar{U}_{\mathrm{L}}$. In our analysis, we shall consider the distinguished regime $b \sim 1$, in which the homogeneous reactions occur in a region with characteristic thickness of the order of the wire spacing.

Equation (4.3) shows different combustion regimes, depending on the wire temperature. For $\left(1-\Theta_{s}\right) \sim 1$, the Arrhenius term is exponentially small and the homogeneous reactions will only be important downstream of the mesh, while in the mesh region only the catalytic reactions will be important. Then, the analysis given before for this region applies, and we may write $-\hat{\Theta}=\hat{Y}=\Psi$, which, in particular, yields a nonzero value for $\hat{Y}_{\infty}$. On the other hand, for values of $\beta_{\mathrm{e}}\left(1-\Theta_{\mathrm{s}}\right) \sim 1$, the effect of the homogeneous reactions cannot be neglected, so that in this case $\hat{Y}=\hat{Y}_{\infty}=0$ far downstream of the mesh.

As previously discussed, when the Peclet number is small, the effect of changes in density and transport properties with temperature can be easily taken into account in the upstream preheated region, while the inner solution remains unchanged if the gas conductivity and the diffusivities are evaluated at the temperature $T_{\mathrm{s}}$. Then, to include the effect of changes in density and transport properties in our formulation, the parameter $b$ has to be substituted by its effective value $b_{\mathrm{s}}=\beta_{\mathrm{e}} P e_{\mathrm{s}}$, defined in terms of the modified Peclet number, $P e_{\mathrm{s}}=\rho_{0} U l /\left(k_{\mathrm{s}} / c_{p}\right)$, and the expression $U_{\mathrm{L}}^{2}=\left(\rho_{\mathrm{s}} / \rho_{0}\right)^{2} 2 L_{\mathrm{F}} D_{T_{\mathrm{s}}} \beta_{\mathrm{c}}^{-2}(1-\phi) Y_{\mathrm{O}_{2} 0} B \exp \left(-E / R T_{\mathrm{e}}\right)$ should be used instead of (4.4) in the definition of $\bar{U}_{\mathrm{L}}=U_{\mathrm{L}} / U$.

\subsection{Solution for $\Theta_{\mathrm{s}}=1$}

We shall begin with an analysis of the steady adiabatic case. As we shall see in section 5, using equation (5.6), under steady and adiabatic conditions the temperature of the wires is equal to the adiabatic flame temperature, so that $\Theta_{\mathrm{s}}=1$. In this case, equation (4.2) yields the relation $\Theta_{\infty}=1-Y_{\infty}$, so that when the homogeneous reaction has gone to completion, $Y_{\infty}=0$, we obtain $T_{\infty}=T_{\mathrm{e}}=T_{0}+Q Y_{\mathrm{F} 0} / c_{p}$.

In this regime, the problem for $-\hat{\Theta}=\hat{Y}$ becomes

$$
\Delta \hat{Y}=\bar{U}_{\mathrm{L}}^{2} \frac{b^{2}}{2} \hat{Y} \exp (-b \hat{Y})
$$

with the boundary conditions $\hat{Y}=\hat{Y}_{a}-x$ at $x \rightarrow-\infty, \hat{Y}=0$ at $x \rightarrow+\infty, \hat{Y}=0$ at $r=\epsilon$, and the lateral symmetry condition $\partial \hat{Y} / \partial y=0$ at $y= \pm \frac{1}{2}$.

Far upstream and downstream of the mesh, the solution becomes one-dimensional, and equation (4.6) can be integrated once to give

$$
\begin{array}{ll}
\frac{\partial \hat{Y}}{\partial x}=-\left[1-\bar{U}_{\mathrm{L}}^{2}(1+b \hat{Y}) \exp (-b \hat{Y})\right]^{1 / 2} & \text { at }-x \gg 1 \\
\frac{\partial \hat{Y}}{\partial x}=-\bar{U}_{\mathrm{L}}[1-(1+b \hat{Y}) \exp (-b \hat{Y})]^{1 / 2} & \text { at } x \gg 1
\end{array}
$$

which allow us to evaluate the diffusive fluxes at the upstream and downstream boundaries of the computational domain, respectively.

The solution to this problem provides the mass of reactant consumed at the wire surface by the catalytic reaction, $m_{1}$, as well as the heat lost from the wire by heat conduction to the 

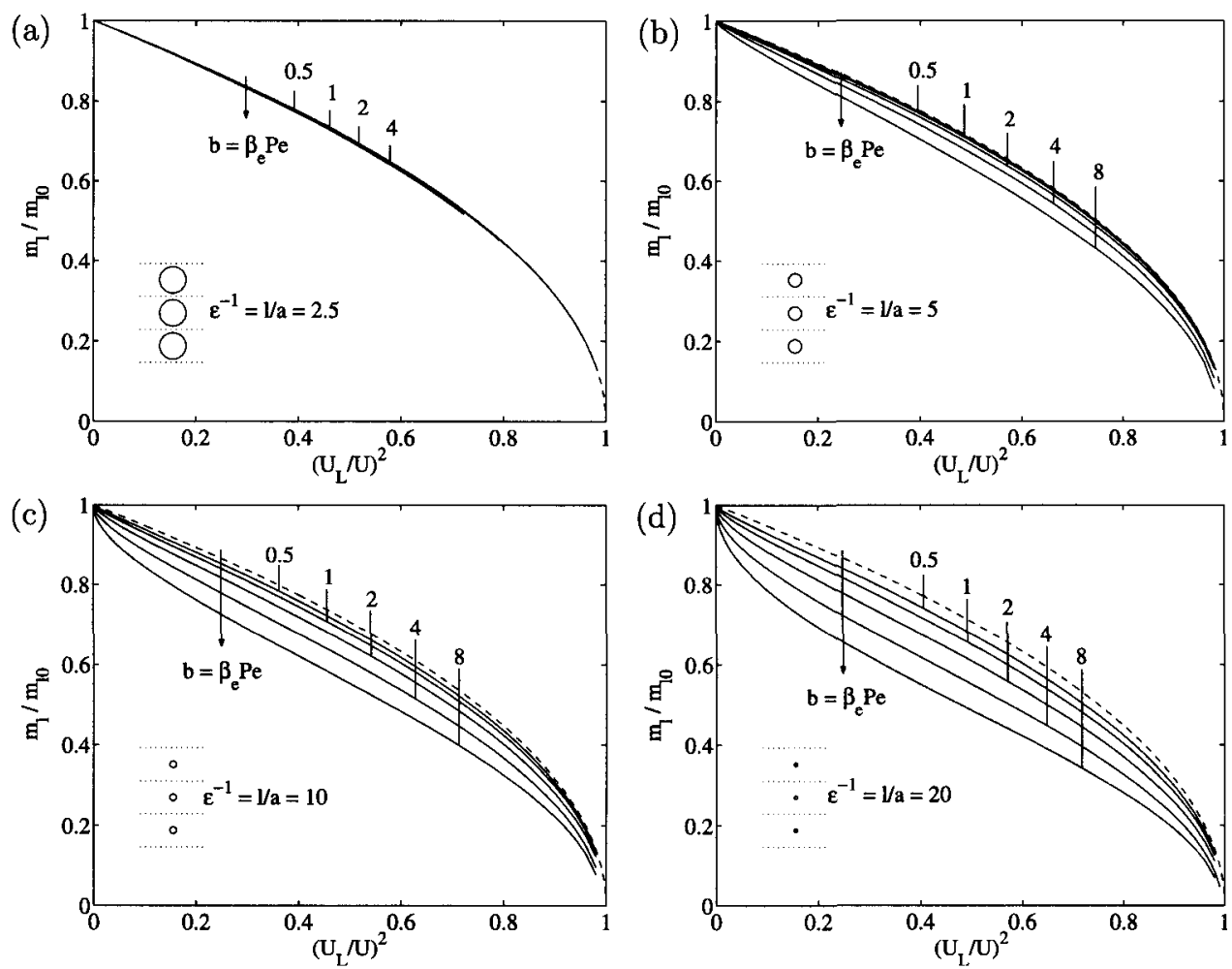

Figure 9. Ratio $m_{1} / m_{10}$ as a function of $\bar{U}_{\mathrm{L}}^{2}$ for different values of $b$ corresponding to $(a) \epsilon^{-1}=2.5$, (b) $\epsilon^{-1}=5,(c) \epsilon^{-1}=10$ and $(d) \epsilon^{-1}=20$, along with the prediction $m_{1} / m_{10}=\left(1-\bar{U}_{\mathrm{L}}^{2}\right)^{1 / 2}$ of the asymptotic analysis for $b=0(---)$. The insets show sketches of the different geometrical configurations.

gas, $q_{1}$, per unit time and unit length of the wire, defined in (2.12) and (2.13), which are given in nondimensional form by

$$
\frac{m_{1}}{m_{10}}=\frac{q_{1}}{Q m_{10}}=\left.2 \pi \epsilon \overline{\frac{\partial \hat{Y}}{\partial r}}\right|_{r=\epsilon}
$$

Equation (4.6) was solved numerically employing a numerical procedure similar to that used for the integration of (3.10). The values of the ratio $m_{1} / m_{10}$ thus obtained are represented in figure 9. The results show that the homogeneous reactions become more and more important as the ratio $\bar{U}_{\mathrm{L}}=U_{\mathrm{L}} / U$ increases, thus reducing the mass of reactant consumed by the catalytic reactions at the wire surface.

In particular, the homogeneous reactions become the only mode of combustion for $\bar{U}_{\mathrm{L}} \geqslant 1$, and when the upstream flow velocity is smaller than the adiabatic laminar flame speed, the nature of the solution changes. Then, we anticipate the existence of a freely propagating premixed flame, detached from the mesh region, travelling towards $x \rightarrow-\infty$ with velocity $U_{\mathrm{L}}-U=\left(\bar{U}_{\mathrm{L}}-1\right) U$ relative to the wires. The flame consumes all the fuel, leaving behind hot products at the adiabatic flame temperature, so that clearly $m_{1}=q_{1}=0$.

4.2.1. Asymptotic solution for $0 \leqslant 1-\bar{U}_{\mathrm{L}}^{2} \ll 1$. For $0 \leqslant 1-\bar{U}_{\mathrm{L}}^{2} \ll 1$, the majority of the fuel is consumed in a gaseous reactive-diffusive region located at distances of order $l$ upstream of the 
mesh. Therefore, the resulting values of the fuel mass fraction in the mesh region are found to be small, of order $\left(1-\bar{U}_{\mathrm{L}}^{2}\right)^{1 / 2}$. Then, the problem for the rescaled variable $\bar{Y}=\left(1-\bar{U}_{\mathrm{L}}^{2}\right)^{-1 / 2} \hat{Y}$, of order unity, reduces to integration of $\Delta \bar{Y}=\left(b^{2} / 2\right) \bar{Y}$ with the boundary conditions $\bar{Y}=0$ at $r=\epsilon, \partial \bar{Y} / \partial x=-\left(1+b^{2} \bar{Y}^{2} / 2\right)^{1 / 2}$ at $-x \gg 1, \partial \bar{Y} / \partial x=-b \bar{Y} / 2^{1 / 2}$ at $x \gg 1$, and the lateral symmetry condition $\partial \bar{Y} / \partial y=0$ at $y= \pm \frac{1}{2}$. Note that in spite of the small values of the fuel mass fraction, which allowed us to linearize the Arrhenius term, the problem remains nonlinear due to the matching condition with the upstream reactive-diffusive region at $-x \gg 1$.

The mass of reactant consumed by the catalytic reactions at the wire surface is then given in a first approximation by

$$
\frac{m_{1}}{m_{10}}=\left.\left.2 \pi \epsilon \overline{\frac{\partial \hat{Y}}{\partial r}}\right|_{r=\epsilon} \simeq\left(1-\bar{U}_{\mathrm{L}}^{2}\right)^{1 / 2} 2 \pi \epsilon \overline{\frac{\partial \bar{Y}}{\partial r}}\right|_{r=\epsilon}
$$

in terms of the average value of the radial derivative of $\bar{Y}$ at the wire surface, to be obtained numerically. However, for small values of $b$ the numerical results are seen to be in good agreement with the asymptotic expression $\left.2 \pi \epsilon \overline{\partial \bar{Y} / \partial r}\right|_{r=\epsilon} \simeq 1-b \Psi_{\infty} / 2^{1 / 2}$.

4.2.2. Staggering effects. The effect of the wire staggering is illustrated in figure 10 . As can be seen, the mass of reactant consumed at the surface of the wires of the first row increases, while that consumed at the wires of the second row decreases, with the staggering distance. On average, however, the amount of reactant consumed by the catalytic reactions remains approximately constant.

\subsection{Solution for $\beta_{\mathrm{e}}\left(\Theta_{\mathrm{s}}-1\right)=\chi_{\mathrm{s}} \sim 1$}

For values of $\beta_{\mathrm{e}}\left(\Theta_{\mathrm{s}}-1\right)=\chi_{\mathrm{s}} \sim 1$ the effect of the homogeneous reactions cannot be ignored, and in a first approximation, equations (4.2) and (4.3) reduce to $-\Theta=\hat{Y}$ and

$$
\Delta \hat{Y}=\bar{U}_{\mathrm{L}}^{2} \exp \left(\chi_{\mathrm{s}}\right) \frac{b^{2}}{2} \hat{Y} \exp (-b \hat{Y})
$$

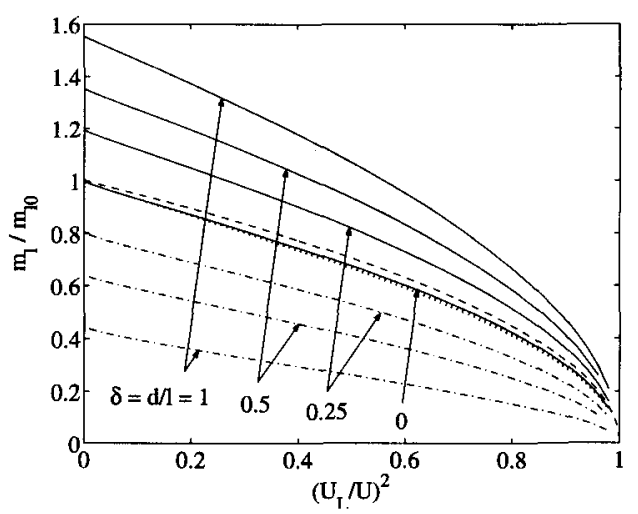

Figure 10. Ratio $m_{1} / m_{10}$ as a function of $\bar{U}_{\mathrm{L}}^{2}$ for the wires of the first row ( $\longrightarrow$ ) and second row (- - - corresponding to $\epsilon^{-1}=10, b=1$ and various staggering distances $\delta$, along with the prediction $m_{1} / m_{10}=\left(1-\bar{U}_{\mathrm{L}}^{2}\right)^{1 / 2}$ of the asymptotic analysis for $b=0(--)$. Although the average value of $m_{1} / m_{10}$ for the first and second rows is also plotted $(\cdots \cdots)$, the curves are almost indistinguishable from the curve corresponding to $\delta=0$. 


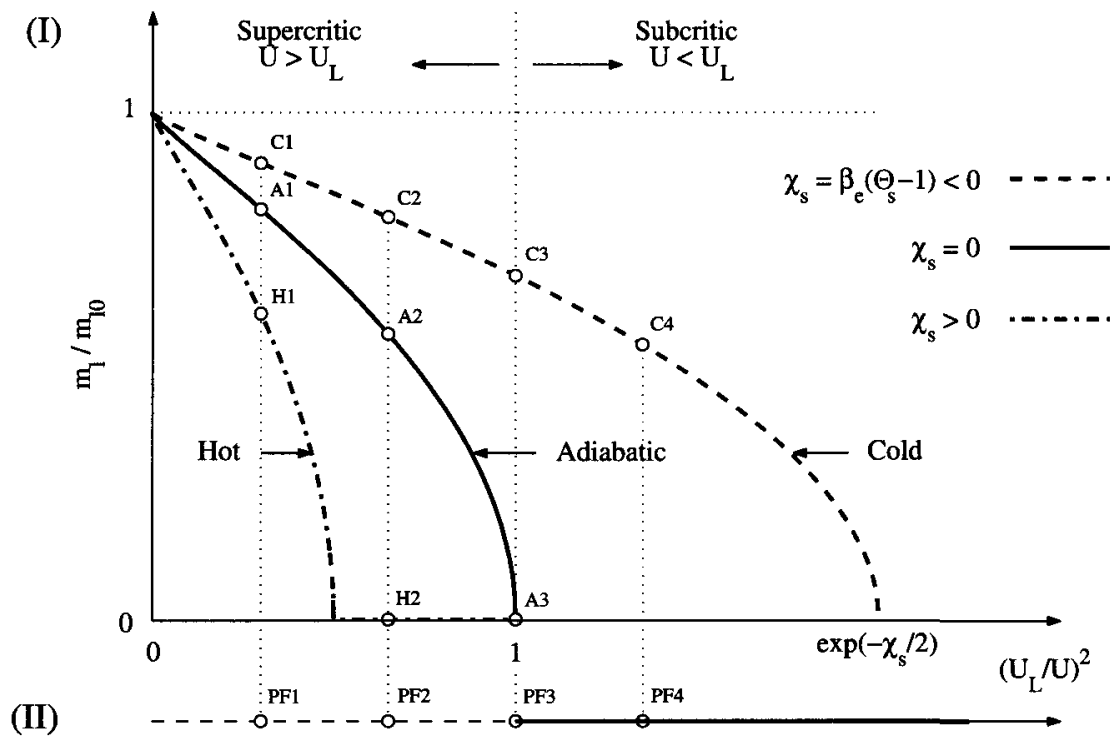

Figure 11. Diagram of combustion regimes. Regime I, reaction zone attached to the wires; regime II, detached premixed flame propagating upstream of the wires towards $x \rightarrow-\infty$ with speed $U_{\mathrm{L}}-U=U\left(\bar{U}_{\mathrm{L}}-1\right)$ relative to the wires.

This equation can be written in the form (4.6) if we substitute the parameter $\bar{U}_{\mathrm{L}}$ by its effective value, $\bar{U}_{\text {Lef }}=\bar{U}_{\mathrm{L}} \exp \left(\chi_{\mathrm{s}} / 2\right)$. Since the boundary conditions for $\hat{Y}$ remain the same, it is clear that the solutions corresponding to $\Theta_{\mathrm{s}}=1+\beta_{\mathrm{e}}^{-1} \chi_{\mathrm{s}} \neq 1$ can be obtained from that corresponding to $\Theta_{\mathrm{s}}=1$ just by rescaling the parameter $\bar{U}_{\mathrm{L}}$ appropriately.

According to this, the values of $m_{1} / m_{10}$ corresponding to $\chi_{\mathrm{s}} \neq 0$ can be obtained, in a first approximation, from those corresponding to $\chi_{\mathrm{s}}=0$ just by rescaling appropriately the horizontal axis of figure 9, leading to the type of results shown in figure 11.

For $\chi_{\mathrm{s}}>0$, i.e. when $\Theta_{\mathrm{s}}>1$ (hot wires), the heterogeneous reactions are enhanced by the high temperature of the wires, reducing the amount of fuel burned by the catalytic reactions at the wire surface, while the opposite is true for $\chi_{\mathrm{s}}<0$, i.e. when $\Theta_{\mathrm{s}}<1$ (cold wires). Consequently, for $\Theta_{\mathrm{s}}<1$ and $1<\bar{U}_{\mathrm{L}}<\exp \left(-\chi_{\mathrm{s}} / 2\right)$, two possible solutions may exist. The upper solution (e.g. C4) corresponds to a reaction zone attached to the wires and the lower solution (e.g. PF4) to a detached premixed flame propagating upstream of the wires towards $x \rightarrow-\infty$ with speed $U_{\mathrm{L}}-U=U\left(\bar{U}_{\mathrm{L}}-1\right)$ relative to the wires. The detached premixed flame solution is no longer stable for $\bar{U}_{\mathrm{L}}<1$ (e.g. PF1) when it is convected downstream by the flow until, eventually, it comes to the mesh region where the reaction zone is stabilized by the additional heat released by the catalytic reactions.

It is interesting to note that, when $\Theta_{\mathrm{s}}>1$ and $\exp \left(-\chi_{\mathrm{s}} / 2\right)<\bar{U}_{\mathrm{L}}<1$, there exists a third regime characterized by the existence of a premixed flame stabilized at distances of order $l / P e$ upstream of the mesh by the heat conducted upstream from the wires (e.g. H2). The heat lost by the wires induces a temperature rise of order $\beta_{\mathrm{e}}^{-1}$ above the adiabatic flame temperature in the reaction zone, which in turn increases the flame propagation velocity by a factor $U / U_{\mathrm{L}}=1 / \bar{U}_{\mathrm{L}} \sim 1$, thus allowing the existence of a premixed flame front propagating with velocity $U>U_{\mathrm{L}}$ with respect to the incoming gases.

The amount of heat lost from the wires by heat conduction to the gas, another important result of the analysis, can be obtained from equation (5.4) of section 5 . 


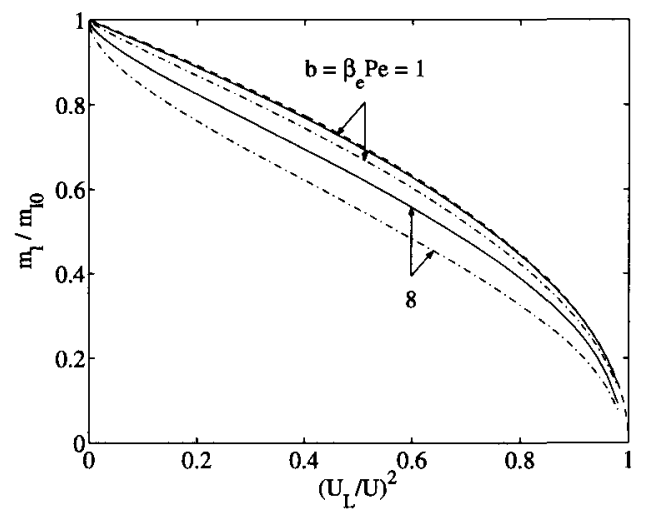

Figure 12. Ratio $m_{\mathrm{l}} / m_{10}$ as a function of $\bar{U}_{\mathrm{L}}^{2}$ for $\epsilon^{-1}=10$ and two different values of $\beta_{\mathrm{e}} P e$ corresponding to stoichiometric mixtures ( - ) and lean mixtures (-. - ), along with the asymptotic prediction $m_{1} / m_{10}=\left(1-\bar{U}_{\mathrm{L}}^{2}\right)^{1 / 2}$ for $b=0(---)$.

\subsection{Analysis for near stoichiometric mixtures}

The formulation given earlier can be easily generalized to include the effect of oxygen consumption when the mixture is close to stoichiometric. For definiteness, we shall consider the case of lean mixtures, $0 \leqslant 1-\phi \ll 1$. In this case, using (4.4) to write the pre-exponential frequency factor in terms of the laminar flame speed retaining the term $2 \phi L_{0} \beta_{\mathrm{e}}^{-1}$, which is now of the same order as $1-\phi$, the leading order problem for $\hat{Y}$ when $\Theta_{\mathrm{s}}=1$ takes the form

$$
\Delta \hat{Y}=\bar{U}_{\mathrm{L}}^{2}\left(\frac{\varphi+b \hat{Y} L_{0}}{\varphi+2 L_{0}}\right) \frac{b^{2}}{2} \hat{Y} \exp (-b \hat{Y})
$$

where $\beta_{\mathrm{e}}(1-\phi)=\varphi \geqslant 0$ is the normalized difference of the equivalence ratio from unity. This equation is to be integrated with the boundary conditions $\hat{Y}=\hat{Y}_{a}-x$ at $x \rightarrow-\infty, \hat{Y}=\hat{Y}_{\infty}$ at $x \rightarrow+\infty$ and $\hat{Y}=0$ at $r=\epsilon$ and the lateral symmetry conditions $\partial \hat{Y} / \partial y=0$ at $y= \pm \frac{1}{2}$. In the two-reactant model, the appropriate nondimensional form of the oxidizer mass fraction in the mesh region is $\hat{Y}_{\mathrm{O}}=\left(Y_{\mathrm{O}_{2}} / Y_{\mathrm{O}_{2} 0}-1+\phi\right) /\left(\phi L_{\mathrm{O}} P e\right)$, which in the case $\Theta_{\mathrm{s}}=1$ verifies $\hat{Y}_{\mathrm{O}}=\hat{Y}$.

The values of $m_{1} / m_{10}$ obtained numerically for stoichiometric mixtures, i.e. $\varphi=0$, are presented in figure 12 as a function of $\bar{U}_{\mathrm{L}}^{2}$ for $\epsilon^{-1}=10$ and two different values of $b$. The results of the one-reactant model are also shown for comparison. Clearly, the amount of fuel burned by the catalytic reactions at the wire surface is more important for stoichiometric mixtures than for lean mixtures, although the qualitative nature of the solution remains unchanged.

Note that far from stoichiometry, i.e. for $0<1-\phi \sim 1$, the factor between brackets in (4.12) is, in a first approximation, equal to one, and therefore the two-reactant model reduces to the one-reactant model discussed in the previous sections.

\section{Transient effects}

Measuring the different terms in (2.11) with the scale $Q m_{10}=Q \rho_{0} U l Y_{\mathrm{F} 0}$, we obtain the nondimensional form of the energy balance per unit time and unit length of wire,

$$
\frac{\partial \Theta_{\mathrm{s}}}{\partial \tau}-\frac{q_{\mathrm{e}}}{Q m_{10}}=\frac{m_{1}}{m_{10}}-\frac{q_{1}}{Q m_{10}}
$$

which governs the time evolution of the nondimensional wire temperature, $\Theta_{s}$. 
In the above equation, time has been nondimensionalized with the characteristic heat-up time of the wire, $t_{\mathrm{w}}=\left(\pi \epsilon^{2} / P e\right)\left(\rho_{\mathrm{s}} c_{\mathrm{s}} / \rho_{0} c_{p}\right) t_{\mathrm{g}}$, defined in terms of the characteristic diffusion time of the gas phase, $t_{\mathrm{g}}=l^{2} / D_{\mathrm{T}}$. In what follows, we shall assume that $t_{\mathrm{w}} / t_{\mathrm{g}}=\left(\pi \epsilon^{2} / P e\right)\left(\rho_{\mathrm{s}} c_{\mathrm{s}} / \rho_{0} c_{p}\right) \gg 1$, so that the unsteady effects associated with the time evolution of the wire temperature can be ignored in the dynamics of the gas phase. In this case, the temperature and concentration fields are given in a first approximation by the quasi-steady problem (4.3), with the parameter $\Theta_{s}$ slowly varying with time according to (5.1).

In the absence of homogeneous reactions, the right-hand side of (5.1) can be easily evaluated from equations (3.22) and (3.23) to give

$$
\frac{m_{1}}{m_{10}}-\frac{q_{1}}{Q m_{10}}=1-\left(Y_{\infty}+\Theta_{\infty}\right)=\left(1-\Theta_{\mathrm{s}}\right)-P e \Psi_{\infty}\left(L_{\mathrm{F}}-\Theta_{\mathrm{s}}\right)+\cdots
$$

an expression that relates the heat lost from the wire by heat conduction to the gas, $q_{1}$, with the mass of reactant consumed by the catalytic reactions, $m_{1}$.

Using (5.2) in (5.1), we obtain the following equation for the nondimensional wire temperature,

$$
\frac{\partial \Theta_{\mathrm{s}}}{\partial \tau}=\tilde{q}_{\mathrm{e}}+\left(1-\Theta_{\mathrm{s}}\right)-P e \Psi_{\infty}\left(L_{\mathrm{F}}-\Theta_{\mathrm{s}}\right)+\cdots
$$

where $\tilde{q}_{\mathrm{e}}=q_{\mathrm{e}} / Q m_{10}$ is the nondimensional external energy addition. Then, by equating the right-hand side of this equation to zero, we obtain the asymptotic temperature reached by the wires for large times, $\Theta_{\mathrm{s}}^{\mathrm{st}}=1+\tilde{q}_{\mathrm{e}}+P e \Psi_{\infty}\left(1+\tilde{q}_{\mathrm{e}}-L_{\mathrm{F}}\right)+\cdots$.

When there are homogeneous reactions, equation (5.2) can be written as

$$
\frac{m_{1}}{m_{10}}-\frac{q_{1}}{Q m_{10}}=1-\Theta_{\infty}=\left(1-\Theta_{\mathrm{s}}\right)-\frac{P e \Psi_{\infty}\left(1-\Theta_{\mathrm{s}}\right)}{\Theta_{\mathrm{s}}}+\cdots
$$

where we have taken into account the fact that in this case $Y_{\infty}=0$, and we have written $\Theta_{\infty}=\Theta_{\mathrm{s}}+P e \Psi_{\infty}\left(1-\Theta_{\mathrm{s}}\right) / \Theta_{\mathrm{s}}+\cdots$ by particularizing (4.2) at $x \rightarrow+\infty$. Note that when the homogeneous reactions are important, equations (2.16) and (2.17) are no longer valid individually, and the first equality in (5.4) emerges from an integral analysis of the equation free from chemistry terms,

$$
P e v \cdot \nabla(\Theta+Y)=\Delta\left(\Theta+\frac{Y}{L_{\mathrm{F}}}\right)
$$

over the fluid volume between two cross sections upstream and downstream of the mesh, taking into account the boundary conditions (2.6)-(2.8) and (2.10), where now $Y_{\infty}=0$, and with the definitions (2.12) and (2.13) of $q_{1}$ and $m_{1}$.

Thus, when there are homogeneous reactions, the equation for the nondimensional wire temperature reads

$$
\frac{\partial \Theta_{\mathrm{s}}}{\partial \tau}=\tilde{q}_{\mathrm{e}}+\left(1-\Theta_{\mathrm{s}}\right)-\frac{P e \Psi_{\infty}\left(1-\Theta_{\mathrm{s}}\right)}{\Theta_{\mathrm{s}}}+\cdots
$$

which under steady conditions yields

$$
\Theta_{\mathrm{s}}^{\mathrm{st}}=1+\tilde{q}_{\mathrm{e}}+\frac{P e \Psi_{\infty} \tilde{q}_{\mathrm{e}}}{1+\tilde{q}_{\mathrm{e}}}+\cdots
$$

so that $\Theta_{s}^{s t}=1$ in the adiabatic case, when there is no external energy addition.

On the other hand, the nondimensional wire temperature may differ from its adiabatic unitary value by an amount of order $P e \sim \beta_{\mathrm{e}}^{-1}$ as soon as the external energy addition, $\tilde{q}_{\mathrm{e}}$, becomes of order $P e$. This is enough to modify the strength of the homogeneous reactions by a factor of order unity. In this case, we recover the regimes of cold and hot wires of figure 11 corresponding, respectively, to positive (energy losses) and negative (energy addition) values of $\chi_{\mathrm{s}}=\beta_{\mathrm{e}}\left(\Theta_{\mathrm{s}}-1\right)=\beta_{\mathrm{e}} \tilde{\mathrm{q}}_{\mathrm{e}} \sim 1$. 


\section{Conclusions}

We have analysed the flow of a reacting mixture past an array of catalytic wires in the limit of small Peclet numbers and large activation energies. We assumed that the catalytic reactions at the wire surface are infinitely fast, so that diffusion is the dominant mechanism in determining the overall rate of the heterogeneous reactions, while we used a one-step overall reaction with Arrhenius kinetics for the gas phase homogeneous reactions.

Three main parameters were found to determine the combustion regime: the ratio, $\bar{U}_{\mathrm{L}}=U_{\mathrm{L}} / U$, of the adiabatic laminar flame velocity to the upstream flow velocity, the product, $b=\beta_{\mathrm{e}} P e$, of the Zeldovich number and the Peclet number, and the nondimensional wire temperature, $\Theta_{\mathrm{s}}=\left(T_{\mathrm{s}}-T_{0}\right) /\left(T_{\mathrm{e}}-T_{0}\right)$.

For $\bar{U}_{\mathrm{L}} \ll 1$, or $1-\Theta_{\mathrm{s}} \sim 1$, the characteristic chemical time of the homogeneous reactions is large compared with the characteristic diffusion time in the mesh region. In this case, the homogeneous reactions are only important downstream of the mesh, while in the mesh region only the catalytic reactions play a role.

The analysis of the catalytic combustion regime provided the values of the temperature and reactant concentrations downstream of the mesh as a function of the Peclet number, the Lewis number of the reactants, the temperature of the wires, and the ratio of the wire radius to the wire spacing, showing that the amount of fuel that leaks through the mesh, as well as the temperature drop from the wire temperature far downstream of the mesh, increases as the radius of the wires decreases and decreases with the staggering distance in the case of a double staggered array.

On the other hand, for $\bar{U}_{\mathrm{L}} \sim 1$ and $\beta_{\mathrm{e}}\left(\Theta_{\mathrm{s}}-1\right)=\chi_{\mathrm{s}} \sim 1$, the characteristic chemical time of the homogeneous reactions, $D_{\mathrm{T}} / \beta_{\mathrm{e}} U_{\mathrm{L}}^{2}$, is of the order of the characteristic diffusion time in the mesh region, $l^{2} / D_{\mathrm{T}}$, and the homogeneous reactions cannot be ignored. In this case, the amount of fuel burned in the gas phase by the homogeneous reactions is seen to grow for increasing values of $\bar{U}_{\mathrm{L}}, b$ and $\Theta_{\mathrm{s}}$, with the homogeneous reactions becoming the only mode of combustion for $\bar{U}_{\mathrm{L}} \exp \left(\chi_{\mathrm{s}} / 2\right) \geqslant 1$.

The various combustion regimes emerging from the previous analysis for different values of the relevant parameters were identified and explained using figure 11.

Several related problems of practical importance remain a topic of further research. The influence of finite rate effects in the catalytic reactions, characterized by the competition between absorption and desorption reactions [12], may come into play for sufficiently low values of the wire temperature, leading to the extinction of the catalytic reactions, thus determining the limits of operation of the system. Finally, for Peclet numbers of order one, complex interactions between the reactive flow and the heterogeneous reactions on the catalytic surface should be expected, including ignition and extinction phenomena of both the homogeneous and heterogeneous reactions [3].

It is worth noting that preliminary results obtained by the integration of the whole set of equations (2.1)-(2.5) for values of the Peclet number of order unity are in very good agreement with the results presented in this paper. The analysis of the flow of a reacting mixture past an array of catalytic wires for Peclet numbers of order unity will be presented in a sequel paper.

\section{Acknowledgments}

Financial support for MV was provided by an FPI grant of the Spanish Consejería de Educación de la Comunidad de Madrid. Part of the work of MV was carried out during a stay at the Institute Henri Poincaré, supported by a Marie Curie Fellowship of the European Community. Financial support for the work of AL has also been provided by the Spanish MCYT under project BFM 
2001-3691. This work was initiated and partially carried out during a stay of AL at Yale University as Adjunct Professor. We would like to acknowledge discussions with Professor Alessandro Gomez of Yale University. The experimental work in his group [2], supported by DARPA, under grant no DAAD19-01-1-0664 (Dr Richard J Paur, Contract Monitor), motivated us to pursue this project. We are also grateful to our colleague Mr Miguel Hermanns for useful discussions on the numerical method.

\section{Appendix A. Low Peclet numbers flow past widely spaced wires}

\section{Appendix A.1. Single layer of wires}

In the limit of widely spaced wires, $\epsilon \ll 1$, the solution of (3.10)-(3.14) is given, in a first approximation, by

$$
\Psi=A-\frac{x}{2}+\Psi_{\mathrm{S}}(x, y)+m \Psi_{\mathrm{D}}(x, y)
$$

where

$$
\begin{aligned}
& \Psi_{\mathrm{S}}(x, y)=\frac{\ln (\cosh 2 \pi x-\cos 2 \pi y)}{4 \pi} \\
& \Psi_{\mathrm{D}}(x, y)=\frac{\partial \Psi_{\mathrm{S}}}{\partial x}=\frac{1}{2} \frac{\sinh 2 \pi x}{\cosh 2 \pi x-\cos 2 \pi y}
\end{aligned}
$$

are, respectively, the solutions corresponding to a linear array of point sources and a linear array of point-source dipoles of unit strength located at $(x, y)=(0, n)$, with $n=0, \pm 1, \ldots$ ([29], p 358). See figure A1 (a) for a schematic representation of the geometrical configuration. In order to guarantee that $\Psi=0$ at $r=\epsilon \ll 1$, we had to add a constant $A$ to the solution in (A.1), where $A$ and $m$ are still to be determined in terms of $\epsilon$.

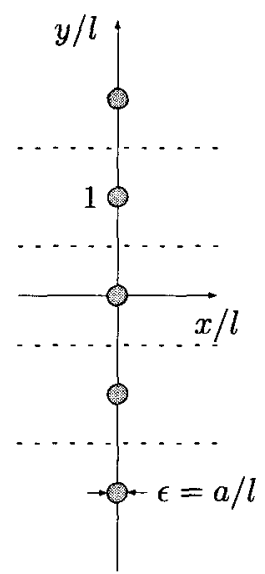

(a)

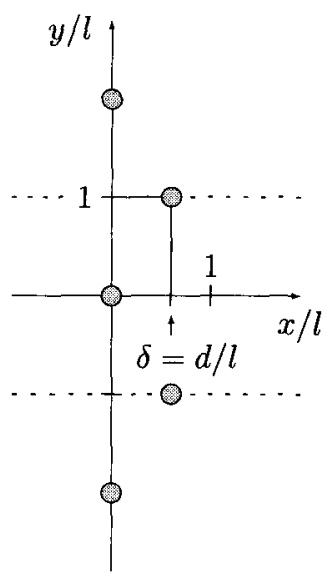

(b)

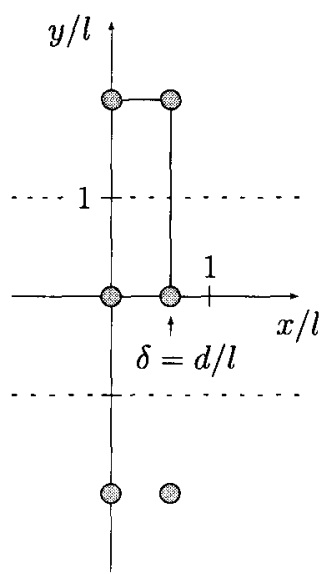

(c)

Figure A1. Geometrical configurations considered in the approximate description of the solution of (3.10)-(3.14) in the limit $\epsilon=a / l \ll 1$. (a) linear array; (b) double staggered array; (c) double aligned array. 
The limiting behaviour of the solution (A.1) far upstream and downstream of the wires is given by

$$
\begin{array}{ll}
\Psi=\Psi_{\infty}=A-\frac{1}{4 \pi} \ln 2+\frac{m}{2} & \text { for } x \gg 1 \\
\Psi+x=\Psi_{a}=A-\frac{1}{4 \pi} \ln 2-\frac{m}{2} & \text { for }-x \gg 1
\end{array}
$$

On the other hand, close to the wire surface, for $r / \epsilon=\tilde{r} \sim 1$, we have

$\Psi=A+\frac{1}{4 \pi} \ln \left[\frac{(2 \pi \epsilon \tilde{r})^{2}}{2}\right]+\left[m\left(\frac{1}{2 \pi \epsilon \tilde{r}}+\frac{\pi \tilde{r} \epsilon}{6}\right)-\frac{\tilde{r} \epsilon}{2}\right] \cos \theta+\mathcal{O}\left(\epsilon^{2}\right)$

where we have written $x=\epsilon \tilde{r} \cos \theta$ and $y=\epsilon \tilde{r} \sin \theta$. Imposing the condition that $\Psi=0$ at $\tilde{r}=1$ up to order $\epsilon$, we obtain

$$
A=\frac{1}{2 \pi} \ln \left(\frac{1}{2 \pi \epsilon}\right)+\frac{1}{4 \pi} \ln 2 \quad m=\frac{3 \pi \epsilon^{2}}{3+(\pi \epsilon)^{2}}
$$

which upon substitution in (A.4)-(A.5) yields

$$
\begin{aligned}
& \Psi_{\infty}=\frac{1}{2 \pi} \ln \left(\frac{1}{2 \pi \epsilon}\right)+\frac{1}{2}\left[\frac{3 \pi \epsilon^{2}}{3+(\pi \epsilon)^{2}}\right] \\
& \Psi_{a}=\frac{1}{2 \pi} \ln \left(\frac{1}{2 \pi \epsilon}\right)-\frac{1}{2}\left[\frac{3 \pi \epsilon^{2}}{3+(\pi \epsilon)^{2}}\right]
\end{aligned}
$$

Finally, from (A.6) and (A.7), we obtain the radial derivative of $\Psi$ at the wire surface giving the diffusive fluxes from and to the wires, namely

$$
\left.\frac{\partial \Psi}{\partial r}\right|_{r=\epsilon}=\left.\frac{1}{\epsilon} \frac{\partial \Psi}{\partial \tilde{r}}\right|_{\tilde{r}=1}=\frac{1}{2 \pi \epsilon}-\cos \theta+\mathcal{O}(\epsilon)
$$

\section{Appendix A.2. Double layer of wires}

In this section, we shall briefly generalize the previous analysis to the case of double layers of wires. In the limit of widely spaced wires, $\epsilon \ll 1$, the solution can be written in a first approximation as

$\Psi=A+\frac{\ln 2}{4 \pi}-\frac{x}{2}+(2-\alpha) \Psi_{\mathrm{S}}^{\mathrm{I}}(x, y)+\alpha \Psi_{\mathrm{S}}^{\mathrm{II}}(x, y)+m^{\mathrm{I}} \Psi_{\mathrm{D}}^{\mathrm{I}}(x, y)+m^{\mathrm{II}} \Psi_{\mathrm{D}}^{\mathrm{II}}(x, y)$

where the fundamental solutions correspond now to arrays of sources and doublets located at the positions of the wires of the first (I) and second (II) rows of wires:

$$
\begin{array}{ll}
\Psi_{\mathrm{S}}^{\mathrm{I}}(x, y)=\frac{\ln (\cosh \pi x-\cos \pi y)}{4 \pi} & \Psi_{\mathrm{D}}^{\mathrm{I}}(x, y)=\frac{\partial \Psi_{\mathrm{S}}^{\mathrm{I}}}{\partial x} \\
\Psi_{\mathrm{S}}^{\mathrm{II}}(x, y)=\frac{\ln [\cosh \pi(x-\delta)-\cos \pi(y-h)]}{4 \pi} & \Psi_{\mathrm{D}}^{\mathrm{II}}(x, y)=\frac{\partial \Psi_{\mathrm{S}}^{\mathrm{II}}}{\partial x}
\end{array}
$$

Now we have to determine the strengths of the sources and doublets, given by the constant $\alpha$, $m^{\mathrm{I}}$ and $m^{\mathrm{II}}$, as functions of $\epsilon, \delta$ and $h$. Note that the constant added to the solution in (A.11) has been written so that $A$ reduces to (A.7) for $h=1$ and $\delta=0$, when the double staggered array reduces to the linear array.

Two different wire-mesh configurations will be considered here, the double staggered array, corresponding to $h=1$, and the double aligned array, corresponding to $h=0$, shown in figures $\mathrm{Al}(b)$ and $(c)$, respectively. In both cases, the number of wires per unit length in the direction transverse to the flow is equal to that of the linear array. 
The limiting behaviour of the solution (A.11) far upstream and downstream of the wires is now given by

$$
\begin{aligned}
& \Psi_{\infty}=\lim _{x \rightarrow+\infty} \Psi(x, y)=A-\frac{1}{4 \pi} \ln 2+\frac{m}{2}-\frac{\alpha \delta}{4} \\
& \Psi_{a}=\lim _{x \rightarrow-\infty} \Psi(x, y)+x=A-\frac{1}{4 \pi} \ln 2-\frac{m}{2}+\frac{\alpha \delta}{4}
\end{aligned}
$$

As discussed before, by imposing the condition that $\Psi=0$ up to order $\epsilon$ at the surface of the wires of the first and second rows, we would obtain a system of four linear relations for the four unknowns $A, \alpha, m^{\mathrm{I}}$ and $m^{\mathrm{II}}$, which would play here the role of equations (A.7) in appendix A.1. Solving this system of equations (whose coefficients could be obtained as explicit functions of $\epsilon, \delta$ and $h$ using symbolic algebra) finally leads to the desired values of $\Psi_{\infty}$ and $\Psi_{a}$ by means of equations (A.14) and (A.15).

Fernández-Pello A C 2002 Micro-power generation using combustion: issues and approaches Proc. Combust. Inst. 29 at press

Kyritsis D C, Guerrero-Arias I, Roychoudhury S and Gomez A 2002 Mesoscale power generation by a catalytic combustor using electrosprayed liquid hydrocarbons Proc. Combust. Inst. 29965

Maruta K, Takeda K, Ahn J, Borer K, Sitzki L, Ronney P D and Deutschmann O 2002 Extinction limits of catalytic combustion in micro-chanels Proc. Combust. Inst. 29 at press

Fu K, Knobloch A, Martinez F, Walther D C, Fernandez-Pello C, Pisano A P, Liepmann D, Miyaska K and Maruta K 2001 Design and experimental results of small-scale rotary engines Proc. ASME 2001 International Mechanical Engineering Congress and Exposition (New York) IMECE2001/MEMS-23924

Waitz I A, Gauba G and Tzeng Y S 2002 Combustors for micro-gas turbine engines ASME J. Fluids Eng. 120109

Lloyd S A and Weinberg F J 1974 A burner for mixtures of very low heat content Nature 25147

Lloyd S A and Weinberg F J 1975 Limits to energy release and utilisation from chemical fuels Nature 257367

Katsui M and Kasegawa T 1998 The science and technology of combustion in highly preheated air Proc. Combust. Inst. 273135

Sitzki L, Borer K, Schuster E, Ronney P D and Wussow S 2001 Combustion in microscale heat-recirculating burners Proc. 3rd Asia-Pacific Conf. on Combustion (Seoul, Korea)

Pfefferle L D and Pfefferle W C 1987 Catalysis in combustion Catal. Rev. Sci. Eng. 29219

Enomoto H, Kato H, Tsue M and Kono M 1998 Catalytic ignition of hydrogen-oxygen on platinum Proc. Combust. Inst. 272259

Treviño C, Liñan A and Kurdiumov V 2000 Autoignition of hydrogen/air mixtures by a thin catalytic wire Proc. Combust. Inst. 281359

Deutschmann O, Schmidt R, Behrendt F and Warnatz J 1996 Numerical modeling of catalytic ignition Proc. Combust. Inst. 261747

Deutschmann O and Schmidt L D 1998 Two-dimensional modeling of partial oxidation of methane on rhodium in a short contact time reactor Proc. Combust. Inst. 272283

O'Connor R P and Schmidt L D $2000 \mathrm{C}_{6}$ oxygenates from $n$-hexane in a single-gauze reactor Chem. Eng. Sci. 555693

Quah E B H, Mathews J F and Li C-Z 2001 Interinfluence between reactions on the catalyst surface and reactions in the gas phase during the catalytic oxidation of methane with air J. Catal. 197315

Karim G A and Kibrya M G 1986 Variations of the lean blowout limits of a homogeneous methane-air stream in the presence of a metallic wire mesh J. Eng. Gas Turb. Power 108446

Schlegel A, Benz P, Griffin T, Weisenstein W and Bockhorn H 1996 Catalytic stabilization of lean premixed combustion: method for improving $\mathrm{NO}_{x}$ emissions Combust. Flame 105332

Ahlström-Silversand A F and Ingemar Odenbrand C U 1997 Thermally sprayed wire-mesh catalysis for the purification of flue gases from small-scale combustion of bio-fuel. Catalyst preparation and activity studies Appl. Catal. A 153177

Ahlström-Silversand A F and Ingemar Odenbrand C U 1999 Modelling catalytic combustion of carbon monoxide and hydrocarbons over catalytically active wire meshes Chem. Eng. J. 73205

Iamarino M, Chirone R, Pirone R, Russo G and Salatino P 2002 Catalytic combustion of methane in a fluidized bed reactor under fuel-lean conditions Combust. Sci. Technol. 174361 
Tamada K and Fujikawa H 1957 The steady two-dimensional flow of viscous fluid at low Reynolds numbers passing through an infinite row of equal parallel circular cylinders Q. J. Mech. Appl. Math. 10423

Miyagi T 1958 Viscous flow at low Reynolds number past an infinite row of equal circular cylinders $J$. Phys. Soc. Japan 13493

Hasimoto $\mathrm{H} 1959 \mathrm{On}$ the periodic fundamental solutions of the Stokes equations and their application to viscous flow past a cubic array of spheres J. Fluid Mech. 5317

Ayaz F and Pedley T J 1999 Flow through and particle interception by an infinite array of closely-spaced circular cylinders Eur. J. Mech. B/Fluids 18173

Wang C Y 2002 Stokes slip flow through a grid of circular cylinders Phys. Fluids 143358

Sangani A S and Acrivos A 1982 Slow flow past periodic arrays of cylinders with application to heat transfer Int. J. Multiphase Flow 8193

Wang W and Sangani A S 1997 Nusselt number for flow perpendicular to arrays of cylinders in the limit of small Reynolds and large Peclet numbers Phys. Fluids 91529

Pozrikidis C 1997 Theoretical and Computational Fluid Dynamics (Oxford: Oxford University Press) 